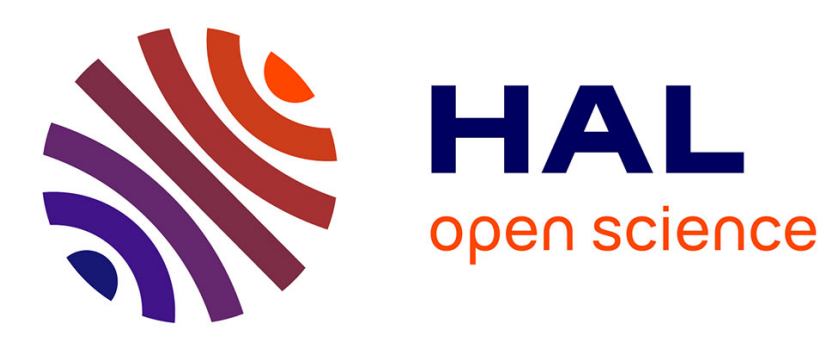

\title{
A Heterogeneous Model of Endovascular Devices for the Treatment of Intracranial Aneurysms
}

\author{
Alain Berod, Christophe Chnafa, Simon Mendez, Franck Nicoud
}

\section{To cite this version:}

Alain Berod, Christophe Chnafa, Simon Mendez, Franck Nicoud. A Heterogeneous Model of Endovascular Devices for the Treatment of Intracranial Aneurysms. International Journal for Numerical Methods in Biomedical Engineering, 2021, pp.e3552. 10.1002/cnm.3552 . hal-03373180

\author{
HAL Id: hal-03373180 \\ https://hal.science/hal-03373180
}

Submitted on 11 Oct 2021

HAL is a multi-disciplinary open access archive for the deposit and dissemination of scientific research documents, whether they are published or not. The documents may come from teaching and research institutions in France or abroad, or from public or private research centers.
L'archive ouverte pluridisciplinaire HAL, est destinée au dépôt et à la diffusion de documents scientifiques de niveau recherche, publiés ou non, émanant des établissements d'enseignement et de recherche français ou étrangers, des laboratoires publics ou privés. 
DOI: $\mathrm{xxx} / \mathrm{xxxx}$

\title{
RESEARCH ARTICLE
}

\section{A Heterogeneous Model of Endovascular Devices for the Treatment of Intracranial Aneurysms}

\author{
Alain Berod*1,2 | Christophe Chnafa ${ }^{2}$ | Simon Mendez ${ }^{1}$ | Franck Nicoud ${ }^{1}$
}

${ }^{1}$ IMAG, Univ Montpellier, CNRS,

Montpellier, France

${ }^{2}$ Sim\&Cure, Montpellier, France

\section{Correspondence}

Alain Berod, Sim\&Cure, Email:

a.berod@sim-and-cure.com

\section{Present Address}

IMAG, UMR 5149 CC 051, Université de

Montpellier, Place Eugène Bataillon, 34095

Montpellier Cedex 5, France

\begin{abstract}
Summary
Numerical computations of hemodynamics inside intracranial aneurysms treated by endovascular braided devices such as flow-diverters contribute to understanding and improving such treatment procedures. Nevertheless, these simulations yield high computational and meshing costs due to the heterogeneity of length scales between the dense weave of the fine struts of the device and the arterial volume. Homogeneous strategies developed over the last decade to circumvent this issue substitute local dissipations due to the wires with a global effect in the form of a pressuredrop across the device surface. However, these methods cannot accurately reproduce the flow-patterns encountered near the struts, the latter strongly dictating the intrasaccular flow environment. In this work, a versatile theoretical framework which aims at correctly reproducing the local flow heterogeneities due to the wires while keeping memory consumption, meshing and computational times as low as possible is introduced. This model reproduces the drag forces exerted by the device struts onto the fluid, thus producing local and heterogeneous effects on the flow. Extensive validation for various flow and geometric configurations using an idealized device is performed. To further illustrate the method capabilities, a real patient-specific aneurysm endovascularly treated with a flow-diverter is used, enabling quantitative comparisons with classical approaches for both intra-saccular velocities and computational costs reduction. The proposed heterogeneous model endeavours to bridge the gap between CFD and clinical applications and ushers in a new era of numerical treatment planning with minimally costing computational tools.
\end{abstract}

\section{KEYWORDS:}

Computational Fluid Dynamics, intracranial aneurysm, endovascular treatment, flow-diverter, heterogeneous modelling, drag model, Immersed Boundary Method

\section{1 | INTRODUCTION}

Intracranial aneurysms (IA) are local deformations of cerebral arteries (see Fig. 17) mostly located in the vicinity of the circle of Willis ${ }^{[1]}$ and harboured by $3 \%$ of the general population ${ }^{[2]}$. A major concern related to this pathology is the rupture of the aneurysmal wall and the subsequent subarachnoid haemorrhage (SAH), which carries high morbidity and mortality rates 4$]$.

\footnotetext{
${ }^{0}$ Abbreviations: CFD: Computational Fluid Dynamics; IA: intracranial aneurysm; IBM: Immersed Boundary Method; FD: flow-diverter; PED: Pipeline Embolization Device
} 


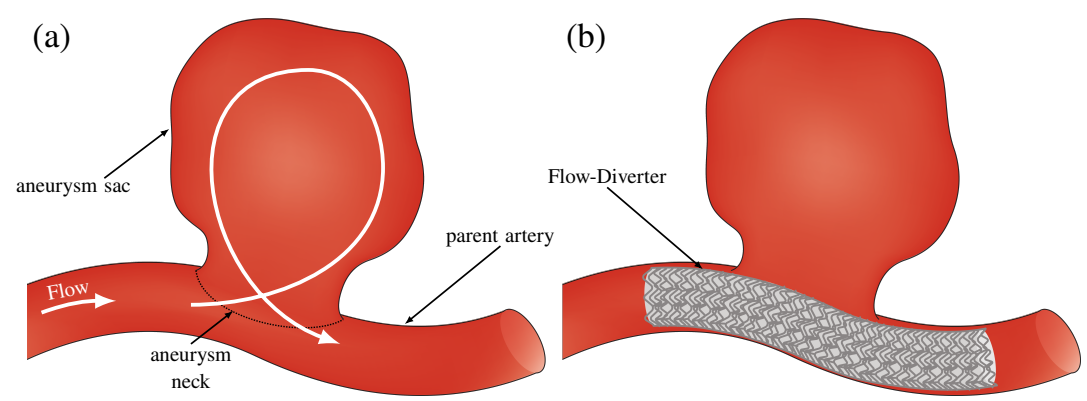

FIGURE 1 Description of saccular aneurysm and Flow-Diverting type of treatment. a: Untreated configuration with glossary related to saccular intracranial aneurysms. Typical flow patterns are exhibited with white arrows. b: Flow-Diverter deployed in the parent artery, intended to reduce the blood flow entrance at the aneurysm's neck. Taken and adapted from ${ }^{[5]}$.

Over the past decades, many treatment modalities for both ruptured and unruptured IA have emerged. Each one of them endeavours to limit the flow in the aneurysm to promote intra-saccular thrombosis, thus occluding and stabilizing the aneurysm. Braided devices such as Flow-Diverters (FDs, see Fig. 1p) are an excellent option in particular to tackle complex aneurysmal geometries (wide neck or small dome-to-neck ratio) ${ }^{[6]}$. As shown in Fig. 1, these devices consist of a very dense weave of thin metallic wires with diameters around $30 \mu \mathrm{m}$, generally made of Nitinol or Cobalt-Chromium ${ }^{6}$. They redirect blood into the parent artery and impede the flow at the aneurysm neck, thus reducing intra-aneurysmal velocities. Additionally, they provide a surface on which neoendothelialisation may occur ${ }^{[7.9]}$, entailing occlusion and potentially volume reduction of the aneurysm, provided that the device is apposed to the arterial wall at proximal and distal positions ${ }^{10]}$, i.e. before and after the neck, respectively. Despite high occlusion and low morbidity and mortality rates ${ }^{[11]}$, unexpected delayed ruptures sometimes occur in approximately $3 \%$ of treated patients $12[13$. Therefore, there is a need to understand all the key mechanisms that can predict chances of success. To this end, intra-saccular hemodynamics has been thought as one of the essential measures that could enhance success predictions 14 . Additionally, hemodynamic information could also be used to minimize the quantity of metal inside patients by studying flow alterations for different devices characteristics. This could help reducing anti-coagulant quantities for patients who underwent endovascular surgical procedures.

To this end, Computational Fluid Dynamics (CFD) has proven to be an invaluable tool enabling to study the effect of device implantation on the blood flow inside patient-specific geometries 14 .17]. To account for deployed braided devices inside arteries, the most 'natural' approach used in most CFD computations is herein referred to as conformal, which removes devices struts from the arterial volume via boolean operations. Conformal methods enable to capture the full complexity of flow-patterns induced by the device presence such as wakes, jetting-flow between wires and wake interactions for very densely packed configurations. Although being considered as a ground-truth approach, this method carries significant drawbacks, mainly due to the large difference of length scales between the wires $(\approx 30 \mu \mathrm{m})$ and the arterial $(\approx 5 \mathrm{~mm})$ diameters. This scale difference implies a high manual meshing cost in order to produce a grid with sufficient quality around the wires. Additionally, these meshes contain a tremendous number of elements, which requires heavy computational resources. These drawbacks are at odds with short time frames needed by both clinicians for treatment planning and manufacturers for new device numerical experiments with many sizes and geometries 18.

To circumvent these issues, a few techniques have emerged over the last decade, which will be herein referred to as homogeneous. The underlying assumption for all these models is that the source of dissipation caused by each device struts produces a collective effect, taking the form of a pressure loss across the surface of the device. Therefore, the sum of local flow dissipations due to the wires can be replaced by a global homogeneous pressure drop to mimic the effect of the device on the fluid. The porous method originally developed by Augsburger et al. ${ }^{[19]}$ and later enhanced by Raschi et al. [20] was the first kind of such homogeneous models. It approximates the device as a porous layer that imposes calibrated pressure losses across its surface via a volume source terms added to the fluid equations. The calibration process of the model was performed by Augsburger et al. [19] thanks to numerical computations on simplified geometries, and was further refined by Raschi et al. [20] who used porous laws through braided meshes. Despite reducing computational costs, Li et al. ${ }^{[21]}$ pointed out that porous model assumptions are not compatible with the very thin structure of endovascular devices. Li et al. ${ }^{[21]}$ went a step further in the modelling of devices by introducing the so-called screen method. This technique aims at faithfully reproducing the flow redirection when passing 
the device while not taking into account each wire individually ${ }^{[22]}$. Nevertheless, the screen approach remains a homogeneous method inherently unable to capture the full complexity of flows around the device struts.

Representing the flow impact of each individual wire at the neck enables to better predict the intra-saccular hemodynamic environment. In this view, several previous CFD studies $[23 \sqrt[26]{26}$ using a conformal approach showed on one hand that local device compaction, and thus struts placement and proximity, is strongly impacting downstream intra-saccular velocities, and on the other hand that device wire density can be optimized to ease treatment decisions for a given case. This strong impact on intrasaccular velocities has been recently confirmed in-vitro by Chodzyǹski et al. [27] who found that by deploying the same FD reference (length and diameter) inside a given idealized geometry three times with the same operator, local pore density changes occurred and caused significant differences in aneurysm filling over the cardiac cycle. One may also anticipate that representing the velocity gradient and associated shear stress at the struts is useful to better represent the platelet activation and thus the capability of the device to promote thrombosis, as described in ${ }^{[26]}$. Nevertheless, these gradients produced by the wires presence are not accessible via homogeneous methods due to both their assumptions and the spatial discretization levels used.

In order to increase the fidelity of CFD for treated aneurysms, a novel heterogeneous model is introduced. This model aims at reproducing both the flow redirection when passing through the device and wakes of the struts by modifying the well-known Immersed Boundary Method (IBM) ${ }^{28}$, to cope with the braided nature of the device. Section 2.1 and Section 2.2 present the core of the method by detailing how the current approach produces localized strut wakes. Validation of the proposed method is presented in Section 3 for both idealized and patient-specific geometries using conformal simulations as ground-truth data for each case.

\section{2 | METHODS}

When being used for CFD computations of endovascularly treated aneurysms, the conformal approach has proven to be robust thanks to the application of no-slip boundary conditions on the nodes of the struts, as depicted in the bottom of Fig. 2 Nevertheless, in order to faithfully capture the geometrical and flow complexities induced by the intertwined wires, the mesh has to be sufficiently refined locally, which introduces at least two major costs: manual-meshing and computational.

To circumvent these issues, homogeneous methods intend to reproduce the local strut dissipation by a global effect in the form of a pressure loss across the device, which is therefore considered as a porous surface as highlighted in the top of Fig. 2 This enables to both remove the strong dependence of the fluid mesh on the local struts geometries and coarsen the mesh to reduce computational costs. Nevertheless, this class of methods carries potential sources of errors due to their underlying assumptions and cannot represent the local flow heterogeneities produced by the wires.

We propose here a heterogeneous model of flow diversion, which is an intermediate approach in terms of computational costs and potential sources of errors, as highlighted in Fig. 2 In this heterogeneous model, the wires forming the endovascular device are replaced by their equivalent 1D neutral fibre. Then, the device is modelled as a collection of linear drag forces $\mathbf{F}$ (force density per unit of length) mimicking the effect of each individual strut on the fluid flow. In this view, the computational mesh is not restricted by the struts, which provides a drastic reduction of both meshing and computational costs. Moreover, each strut is modelled by its corresponding drag force, so that the flow heterogeneities downstream of the device (wakes and jet-like regions) are conserved. These drag forces are applied on fluid regions, highlighted in grey in the middle of Fig. 2, which can be larger than the struts.

To faithfully mimic the local effect of the struts on the flow without meshing them, the proposed heterogenous model relies upon the paradigm of Immersed Boundary Method (IBM) ${ }^{28}$. IBM is used to represent fluid-structure interactions without explicitly meshing the interface between the fluid and solid domains. Instead, the fluid-structure coupling is performed via a volume source term added to the Navier-Stokes equations so that the fluid flow can feel the presence of the solid domain. This writes:

$$
\begin{aligned}
& \rho\left(\frac{\partial \mathbf{u}}{\partial t}+\nabla \cdot(\mathbf{u} \otimes \mathbf{u})\right)=-\nabla p+\nabla \cdot \overline{\bar{\tau}}+\mathbf{f} \\
& \boldsymbol{\nabla} \cdot \mathbf{u}=0
\end{aligned}
$$

with $\mathbf{u}$ the fluid velocity, $\rho$ the density, $p$ the pressure and $\overline{\overline{\boldsymbol{\tau}}}$ the shear stress tensor. In the current approach, $\mathbf{f}$ stands for the volume source term intended to mimic the impact of the device on the flow. Its value is zero everywhere except in the vicinity of the solid parts, i.e. the wires of the endovascular device. 


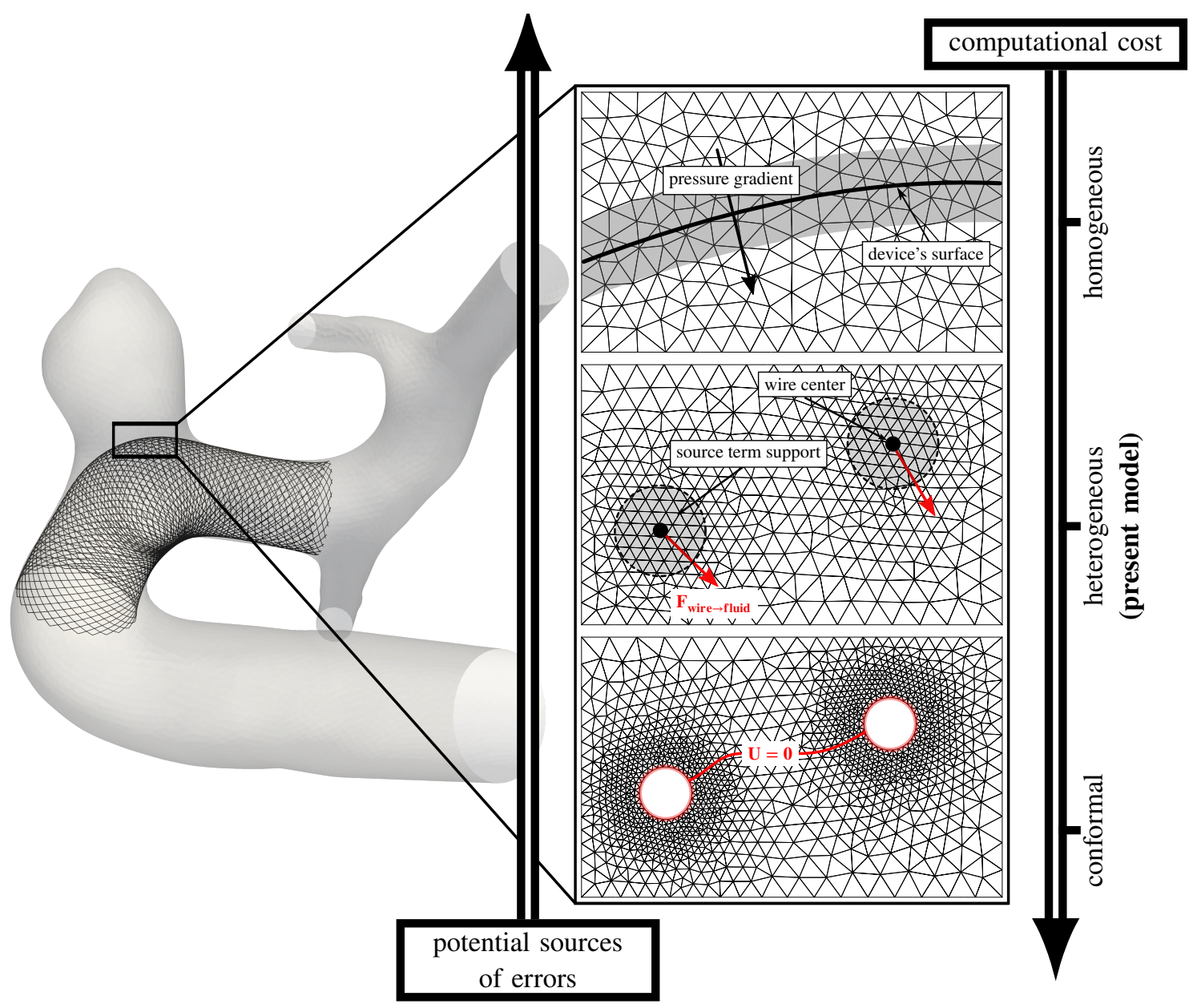

FIGURE 2 Overview of CFD modelling strategies for FD-treated aneurysms. When considering costs and potential sources of errors, the proposed method is lying between homogeneous paradigms such as porous models ${ }^{[19]}$ or the screen approach ${ }^{[22]}$ and more classical techniques herein referred to as 'conformal' [14]. It aims at reproducing the impact of the wires on the flow via a hydrodynamic force $\mathbf{F}_{\text {wire } \rightarrow \text { fluid }}$ (in red) regularized onto a localized volume source term region (in grey) on the fluid mesh.

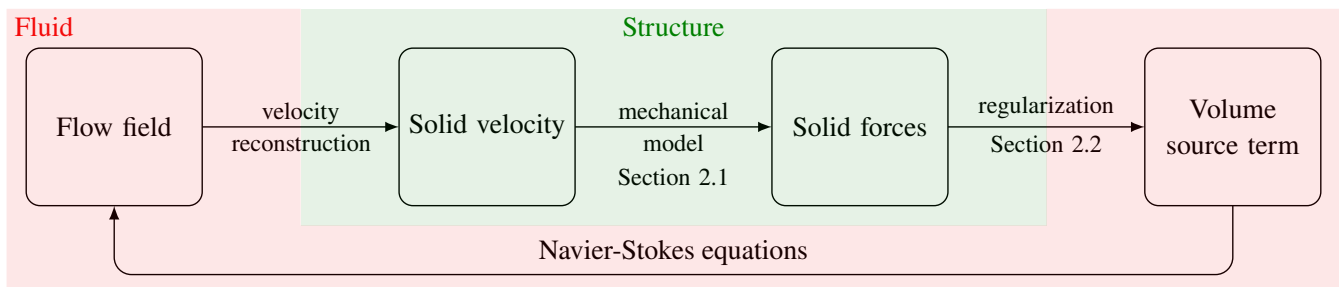

FIGURE 3 Main principles of the Immersed Boundary Method (IBM). Steps involving fluid and solid parts are highlighted in red and green, respectively. The sections involving the main developments are indicated for the ease of comprehension.

Fig. 3 presents the main ingredients of the Immersed Boundary Method ${ }^{28]}$ which is the foundation of the proposed model. Starting from a fluid velocity field, the velocity reconstruction step enables to give to the solid parts local velocity information which in turns is used to model the force generated by the solid onto the fluid. Then, in order for the flow to feel the presence of those forces, a regularization step adds a volume source term in the fluid Navier-Stokes equations. Solving these equations with this newly added source term finally impacts the original velocity field, therefore providing a coupling between fluid and solid. 
For the purpose of the intended applications presented herein, only the key steps associated with the most important development efforts will be described in dedicated sections (see Fig. 3). The remaining steps do not have specific sections associated with them but are still tackled in the present manuscript.

These developments based upon IBM were implemented in the in-house finite-volume YALES2BIO CFD solver https://imag. umontpellier.fr/ yales2bio/index.html Inheriting massive parallel capabilities from the YALES2 software ${ }^{29}$, YALES2BIO intends to solve blood related problems at both microscopic ${ }^{[30 \mid 35]}$ and macroscopic scales ${ }^{[36}{ }^{369}$. Extensive validations have been performed over the years, as demonstrated in the aforementioned references. A pressure-projection method is used to handle the incompressibility constraint Eq. 1b which leads to solving a Poisson equation for the pressure using a Deflated Preconditioned Conjugated Gradient solver $\stackrel{40}{40}$. Minimally dissipative fourth-order spatial and time discretization schemes are used to explicitly advance the velocity field. More details on the numerical method of YALES2 can be found in 29.

\section{1 | Cylinders forces}

As already stated, the current model replaces the struts volumes by their 1D neutral fibre, which is equivalent to using the beam theory in the field of solid mechanics. Therefore, it is considered that each of these 1D struts applies drag forces $\mathbf{F}$ onto the fluid in their close vicinity while being static with respect to the flow. Focus is now made on the computation of these forces for each edge of the endovascular device.

\subsection{1 | Working assumptions}

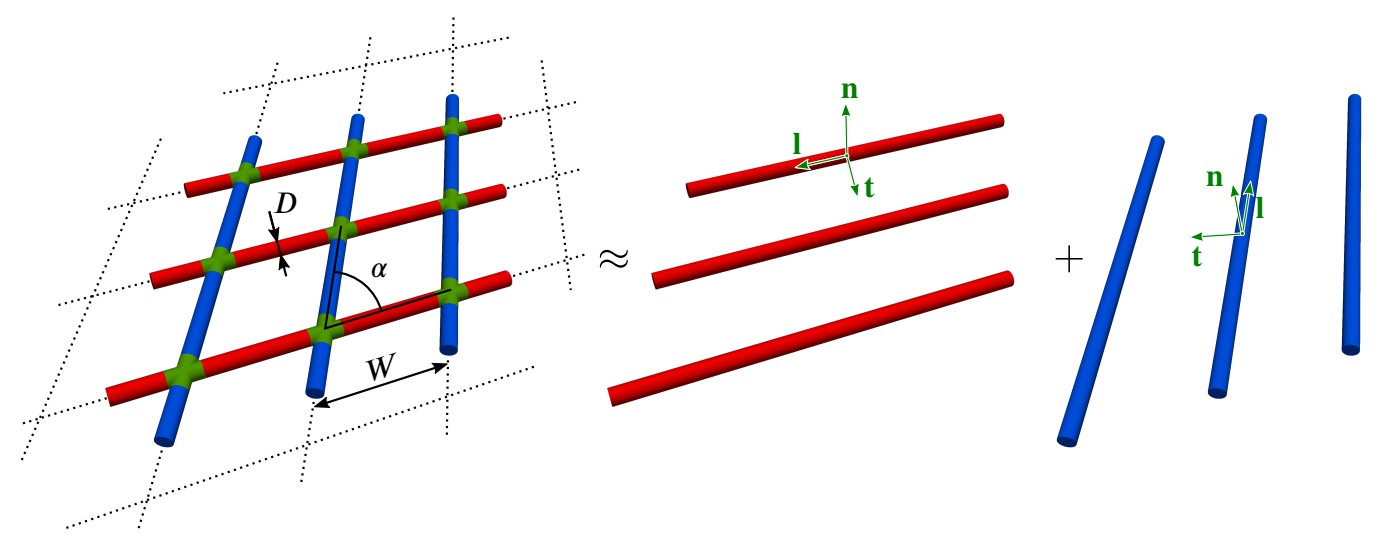

FIGURE 4 The device is assumed to consist of a superposition of two independent families of infinite parallel cylinders (red and blue), which entails that the blue wires are not affected by the flow redirection due to the red ones, and vice-versa. Crossings parts (in green) are also neglected. The device is fully parametrized by the inter-wire distance $W$, the wire diameter $D$ and the small inner angle $\alpha$. Attached to each cylinder, a local basis (in green) is used to decompose drag forces into normal $\mathbf{n}$, tangential $\mathbf{t}$ and longitudinal $\mathbf{I}$ directions. It should be noted that the $\mathbf{t}$ unit vector is located in the plane built by all the wires.

This study focuses on braided endovascular devices consisting in two families of crossing struts (see Fig. 4). Three parameters depicted in Fig. 4 are used to fully characterize the device geometry: the inter-wire distance $W$, the strut diameter $D$ and the acute angle formed at the intersections between the struts, $\alpha$. Typical values of those parameters encountered for Pipeline Embolization Device (PED) flow-diverters are available in ${ }^{41}$. Attached to each cylinder, a local basis can be built as depicted in the right of Fig. 4 . It consists of normal, tangential and longitudinal unit vectors, denoted $\mathbf{n}, \mathbf{t}$ and $\mathbf{l}$, respectively. $\mathbf{n}$ is normal to the device, $\mathbf{I}$ is aligned with the strut and $\mathbf{t}$ is tangential to the device (not the strut) and normal to $\mathbf{n}$ and $\mathbf{l}$.

In order to build the drag model needed to obtain the force $\mathbf{F}_{\mathbf{m}}$ for each $\mathrm{m}^{\text {th }}$ edge of the device, the following assumptions have been made:

H1 The local curvature of the wires, as well as the intertwining are neglected. This amounts to considering straight merged cylinders as schematized to the left of Fig. 4 
H2 It will prove useful to restrict to situations where the inter-wire distance $W$ is much larger than the strut diameter $(W / D>5$, say). This assumption is not very restrictive and is reasonably met by flow-diverters and intra-saccular types of devices extensively listed in 6 .

H3 The inter-wire distance $W$ is assumed to be very small compared to the typical size of the aneurysm neck so that edge effects do not control the flow over the major part of the device. Therefore, from a modeling point of view, the device is considered to be immersed within an infinite fluid domain. Note that edge effects are partly accounted for, as the flow velocity near the edges of the neck is strongly slowed down by the presence of the walls of the artery and of the aneurysm.

H4 The device struts are divided into two distinct 'families' made of parallel infinite cylinders, as described to the right of Fig. 4 Interactions between cylinders are restricted to their parallel family members, which means, using the color code of Fig. 4, that the flow deviation caused by blue wires is not taken into account when computing drag forces applied by red wires onto the fluid, and vice versa. Parallel family interactions will be further referred to as 'intra-familial' interactions in the remaining of the paper, as opposed to 'extra-familial' ones.

H5 The locations where cylinders merge together (in green) herein referred to as 'crossings' are represented by the superposition of the drag forces related to the two families of struts, without further modelling effort.

Hypotheses from $\mathrm{H} 1$ to $\mathrm{H} 3$ are 'geometrical' since they only depend on the geometric characteristics of endovascular devices. H4 and H5 are 'modelling' hypotheses because they are used to construct the drag forces models at the struts. In view of these hypotheses, it is assumed that intra-familial interactions between cylinders are negligible in the longitudinal direction $\mathbf{l}$ when compared to the ones present in the normal-tangential plane $(\mathbf{n}, \mathbf{t})$. It is equivalent to state that there is an invariance with respect to the longitudinal direction. This means that the problem reduces to a 2D problem: how does one array of aligned 2D cylinders interact with each other and what is the resulting drag force applied on the fluid ? Even though the longitudinal direction $\mathbf{l}$ is not taken into account in cylinders interactions, drag forces are still present in this direction and will be described later.

In the remainder of this section, it is assumed that cylinders are immersed in a known, uniform velocity field denoted $\mathbf{U}_{\infty}$. Given this upstream velocity $\mathbf{U}_{\infty}$, we propose a model for the drag forces in the directions $\mathbf{n}, \mathbf{t}$ and $\mathbf{l}$, on the basis of the assumptions $\mathrm{H} 1-\mathrm{H} 5$, which is a classical way of building drag models. The upstream Reynolds number defined by $\operatorname{Re}=\frac{\left\|\mathbf{U}_{\infty}\right\| D}{v}$ with $v$ the fluid kinematic viscosity and $D$ the diameter of the wires is sufficient to characterize the drag force experienced by an isolated 2D cylinder. For an array of 2D parallel cylinders, another non-dimensionalized parameter measuring the closeness of cylinders, the $\tilde{W} / D=\frac{W \sin \alpha}{D}$ ratio, say, has to be introduced (see Fig. 4 .

Relevant ranges for Re and $\tilde{W} / D$ have to be considered to make sure that the outcome of the modelling effort will indeed be useful to biomedical applications. From previous observations and computations, the highest Re values encountered in FDtreated intracranial aneurysms is approximately $R e=20$. This is confirmed by Raschi et al. $\stackrel{\text { [20] }}{~ w h o ~ s t a t e s ~ t h a t ~ t h e ~ h i g h e s t ~ R e ~ i s ~}$ usually "around $R e=20$, hardly exceeding 60 but still falling in the transition zone between low and intermediate Re". During the whole cardiac cycle, endovascular wires can experience both low and high Reynolds numbers depending on their position at the neck. The high Reynolds regimes mainly drive the most important intra-saccular flow features such as large recirculation regions and incoming jets. Since the ability of endovascular devices to reduce intra-saccular velocities and increase residence time is critical for high Reynolds flow regimes, i.e. when convective effects are dominant, the current modelling effort focuses on these regimes. A heterogeneous model which would include both low and high Reynolds is of course very desirable and could be obtained by combining a model for the diffusive regime with the one proposed in this work. For the remainder of the paper, we are concerned with modelling the flow through a weaving of wires in the high Reynolds regimes, i.e. when convection effects dominate. The performances of the model when the Reynolds number gets small will be assessed for the sake of completeness. Regarding the $\tilde{W} / D$ ratio, its values range approximately from 6 to 10 for PEDs flow-diverters according to the data available in 41 .

From the local basis defined in Fig. 4 , the drag force $\mathbf{F}_{\mathbf{m}}$ exerted by the fluid on each cylinder is decomposed into its normal $F_{n}$, tangential $F_{t}$ and longitudinal $F_{l}$ components:

$$
\mathbf{F}_{\mathbf{m}}=F_{t} \mathbf{t}+F_{n} \mathbf{n}+F_{l} \mathbf{l}
$$


As a note of caution regarding units, it should be noted that forces per unit of length are considered for drag forces $\mathbf{F}_{\mathbf{m}}$, since only 2D cylinders are studied. The local orthonormal basis $(\mathbf{n}, \mathbf{t}, \mathbf{I})$ is built to ensure that the scalar product of each unit vector with $\mathbf{U}_{\infty}$ is positive. Reynolds numbers associated with each velocity components are then computed as:

$$
\left\{\begin{array}{l}
\operatorname{Re}_{n}=\frac{\left(\mathbf{U}_{\infty} \cdot \mathbf{n}\right) D}{v}, \\
\operatorname{Re}_{t}=\frac{\left(\mathbf{U}_{\infty} \cdot \mathbf{t}\right) D}{v}, \\
\operatorname{Re}_{l}=\frac{\left(\mathbf{U}_{\infty} \cdot \mathbf{l}\right) D}{v} .
\end{array}\right.
$$

As previously stated, the drag force is assumed to be invariant in the longitudinal direction, so that the 3D problem is equivalent to that of an infinite series of cylinders aligned with the tangential direction as schematized in Fig. 5

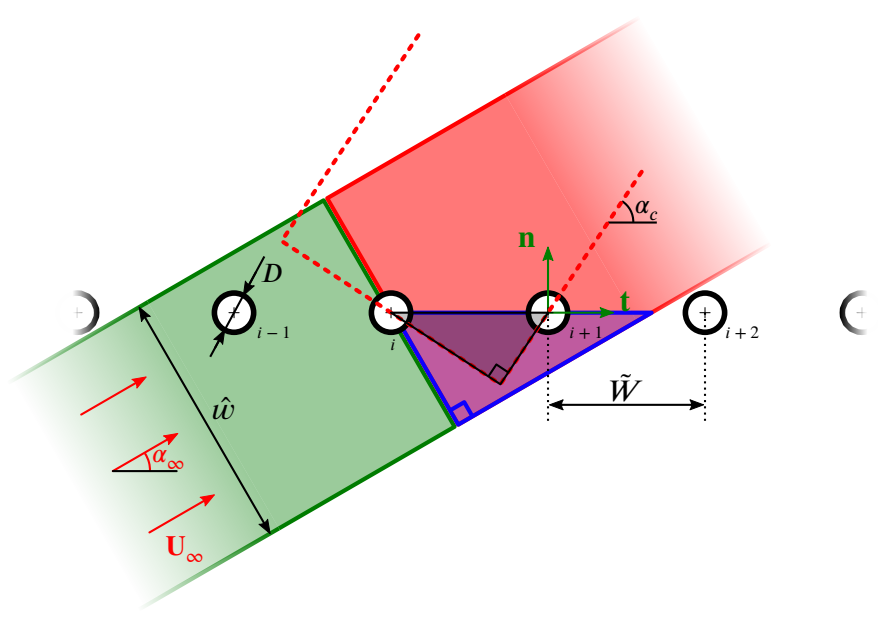

FIGURE 5 An infinite single array of aligned cylinders of diameter $D$ and inter-wire distance $\tilde{W}$ is submitted to an upstream undisturbed velocity field $\mathbf{U}_{\infty}$; the angle of attack is $\alpha_{\infty}$. The total force exerted on each cylinder by the fluid can be decomposed in the normal-tangential basis (in green) denoted by $(\mathbf{n}, \mathbf{t})$. Green and red regions of width $\hat{w}$ aim to represent portions of the fluid influencing and being influenced by the $i^{\text {th }}$ cylinder, respectively. In the case displayed here, it is considered that the $i^{\text {th }}$ cylinder only influences one cylinder in its downstream wake, the $(i+1)^{\text {th }}$ cylinder and that it is directly influenced by the wake of a single cylinder, the (i-1) ${ }^{\text {th }}$ one. Additionally, the critical angle $\alpha_{c}$ which corresponds to the case where the regions' edges lie in the middle of the following and preceding cylinders is depicted with red dotted lines. It is considered that the condition $\alpha_{\infty}<\alpha_{c}$ entails cylinders wake interactions and thus drag forces modifications compared to the isolated cylinder case.

\subsection{2 । Normal term $F_{n}$}

The normal component of the force applied by an array of cylinders subjected to a purely normal incoming flow has already been studied by Müller et al. [42], who proposed an empirical fit using a combination of existing analytical drag models and numerical computations. When compared with CFD results, this model provides a maximum error below $4 \%$ for the ranges $0<\operatorname{Re}_{n}<20$ and $2<\tilde{W} / D<20$, which encompasses our intended ranges of interest. Following Müller et al. ${ }^{[42]}$, the normal component $F_{n}$ is given by:

$$
F_{n}\left(\operatorname{Re}_{n}, \tilde{W} / D\right)=C_{D} \frac{\rho\left(\mathbf{U}_{\infty} \cdot \mathbf{n}\right)^{2} D}{2}
$$


with $\rho$ the fluid density and $C_{D}$ the so-called dimensionless drag coefficient that depends on the normal Reynolds number $\operatorname{Re}_{n}$ and the $\tilde{W} / D$ ratio. Following Müller et al. ${ }^{[42]}$, its expression writes:

$$
C_{D}=C_{D, M}\left(1+1.966 \mathrm{Re}_{n}^{0.4193} \times \exp \left[-8.774\left(\frac{\tilde{W}}{D}\right)^{-0.3143} \mathrm{Re}_{n}^{-0.4688}\right]\right),
$$

with $C_{D, M}$ being the drag coefficient analytically derived by Miyagi ${ }^{[43]}$ for Stokes flow regime $(\operatorname{Re} \rightarrow 0)$ and $1.43<\tilde{W} / D<$ 100 ranges. Its expression is given by:

$$
\left\{\begin{aligned}
C_{D, M} & =\frac{16 \pi a_{0}}{\operatorname{Re}_{n}}, \\
a_{0} & =\left[1-2 \ln (2 \kappa)+\frac{2}{3} \kappa^{2}-\frac{1}{9} \kappa^{4}+\frac{8}{135} \kappa^{6}-\frac{53}{1350} \kappa^{8}+\frac{1112}{42525} \kappa^{10}-\frac{241643}{13395375} \kappa^{12}+\frac{18776}{1488375} \kappa^{14}\right]^{-1}, \\
\kappa & =\frac{\pi D}{2 \tilde{W}} .
\end{aligned}\right.
$$

It should be noted that this expression induces that for a given Reynolds number, the drag force increases when the $\tilde{W} / D$ ratio decreases. Moreover, it is worth mentioning that this component only depends on the normal Reynolds $\operatorname{Re}_{n}$.

\subsection{3 | Tangential term $F_{t}$}

To the best of the authors' knowledge, no modelling effort dedicated to tangential drag forces for an array of equally-spaced cylinders is reported in the literature, at least for the ranges of Reynolds number and $\tilde{W} / D$ ratio of interest. For example, Crowdy [44] focused on an inviscid and irrotational flow, which is not relevant to our configuration. This led us to build such a tangential drag model using both CFD computations and existing drag models.

Considering an infinite series of cylinders as depicted in Fig. 5 the first step is to consider the case where $\tilde{W} / D \rightarrow \infty$, meaning that cylinders are isolated and do not interact with each other. We also consider a purely tangential incoming flow. Then, the drag force in the tangential direction can be computed using the so-called universal drag model derived by Marheineke and Wegener [45], applicable to any Reynolds number and incident flow angle, except for purely longitudinal configuration. Marheineke and Wegener ${ }^{[45]}$ fitted CFD results with existing drag laws at various Reynolds regimes to obtain their continuous model.

Note that the normal unit vector $\mathbf{n}$ in ${ }^{45]}$ corresponds in our case to the tangential one $\mathbf{t}$. By using our notation convention, the tangential drag force term for a unique isolated cylinder denoted in the following by $F_{t}^{0}$ is given by:

$$
F_{t}^{0}=\frac{\rho v^{2}}{D} \operatorname{Re}_{t}^{2} C_{t}\left(\operatorname{Re}_{t}\right)
$$

with $C_{t}$ being the dimensionless drag coefficient in the tangential direction. It should be noted that it only depends on the tangential Reynolds number $\operatorname{Re}_{t}$ and is given by:

$$
C_{t}\left(\operatorname{Re}_{t}\right)=\exp \left[\sum_{j=0}^{3}\left(p_{t, j}\left(\ln \operatorname{Re}_{t}\right)^{j}\right)\right]
$$

with $p_{t, 0}=1.6911, p_{t, 1}=-6.722 \times 10^{-1}, p_{t, 2}=3.3287 \times 10^{-2}$ and $p_{t, 3}=3.5015 \times 10^{-3}$. Eq. 8 is valid in the interval $\operatorname{Re}_{t}=[0.1,100]$, which encompasses the intended range of interest relevant to the proposed heterogeneous model. It should be noted that the normal force given by Eq. 4 approaches Eq. 7 on the conditions that $\tilde{W} / D \rightarrow \infty$ and that the corresponding Reynolds numbers are equal.

The second step aims at taking into account the interactions between cylinders. The main idea is that due to the angle of attack $\alpha_{\infty}$, the flow seen by a cylinder may result from the interaction with a different number of cylinders. We first consider a finite series of $N_{c}$ cylinders aligned in the tangential direction. Each of them is labelled with an index $i$ going from 0 to $N_{c}-1$, following the order defined by the direction of the tangential velocity as in Fig. 5 They are separated by an inter-wire distance $\tilde{W}$ and it is hypothesized that they are subjected to a purely tangential incoming flow. Since the infinite assumption is no more relevant in this case (due to the proximity of the cylinders), each cylinder experiences a drag force which is different than its neighbours. More specifically, it is postulated that the wakes of the cylinder induce a reduction of the drag forces 
downstream, that is $F_{t}^{i+1} / F_{t}^{i}<1$. Additionally, it is assumed that the rate of decrease is constant for each cylinder, implying that $F_{t}^{i+1}=q F_{t}^{i}, \forall i \in\left[0, N_{c}-1\right]$ with $0<q<1$. Consequently, this amounts to considering that the force exerted by each cylinder on the fluid follows a geometric sequence with a common ratio $q$ to be determined later. In order to homogenise the global effect of those $N_{c}$ cylinders onto the fluid, one can consider that each cylinder applies a force $F_{t}$ equals to the average of the total force exerted by all the interacting cylinders such that:

$$
F_{t}=\frac{1}{N_{c}}\left(\sum_{j=0}^{N_{c}-1} F_{t}^{j}\right)=\frac{1}{N_{c}}\left(\frac{F_{t}^{0}\left(1-q^{N_{c}}\right)}{1-q}\right),
$$

where $F_{t}^{0}$ is the drag force experienced by the first cylinder. Since it does not have any upstream counterpart, it is considered to be isolated and thus, its drag force expression is given by Eq. 7

The last step aims at obtaining an expression of the drag force exerted by one cylinder in a infinite array when the incoming flow is not purely tangential, as depicted by Fig. 5 Starting from Eq. 9, the number of cylinders that are interacting $N_{c}$ and the geometric common term $q$ are the two main unknowns. To derive an expression for $N_{c}$, two areas coloured in green and red in Fig. 5 are defined and are oriented by the angle of attack $\alpha_{\infty}$. Since $\mathbf{n}$ and $\mathbf{t}$ are oriented according to $\mathbf{U}_{\infty}, \alpha_{\infty}$ lies in the [0, $\left.\pi / 2\right]$ range. The red/green areas correspond to the domain of influence of the $i^{\text {th }}$ cylinder and the zone influencing the drag at the same obstacle, respectively. They extend up to infinity parallel to the incoming flow direction and have a finite perpendicular width $\hat{w}$, expressed as a linear function of the wire diameter $D$ such that $\hat{w}=N_{w} D, N_{w}$ being the number of cylinder diameter across the influencing region (see Fig. 5p. When reaching a critical angle of attack $\alpha_{c}$, the edge of the green region intersects the center of (i-1) ${ }^{\text {th }}$ cylinder, as shown in Fig. 5 (see the red dotted line). Therefore, the condition $\alpha_{\infty}>\alpha_{c}$ entails no cylinder interactions and thus $N_{c}=1$ since the coloured regions do not overlap downstream and upstream cylinders. On the other hand, $\alpha_{\infty} \leq \alpha_{c}$ implies that the $\mathrm{i}^{\text {th }}$ cylinder is influenced and influences $N_{c}>1$ cylinders upstream and downstream respectively. It is expected that $N_{c}$ should increase with decreasing $\alpha_{\infty}$ to reflect that interactions are getting stronger as more cylinders are added in the influenced region.

Following previous considerations, $N_{c}$ can be computed such that:

$$
N_{c}=\left\{\begin{array}{cc}
1 & \text { if } \alpha_{\infty}>\alpha_{c}, \\
\frac{\sin \alpha_{c}}{\sin \alpha_{\infty}} & \text { otherwise. }
\end{array}\right.
$$

The expression on the second line of Eq. 10 has been chosen so as to obtain $N_{c}=1$ if the edge of the influencing region touches the $(\mathrm{i}+1)^{\mathrm{th}}$ cylinder center, i.e. when $\alpha_{\infty}=\alpha_{c}$. For smaller $\alpha_{\infty}$ angles, this expression compares the length of the hypotenuses of the black and blue rectangular triangles in Fig. 5. It should be noted that $N_{c}$ is no more an integer when cylinders interact due to the previous definition and that it is a function of both $\alpha_{\infty}$ and $\tilde{W} / D$. Plugging Eq. 10 into Eq. 9 entails that without cylinder interactions, i.e. $N_{c}=1$, the drag force exerted by the $\mathrm{i}^{\text {th }}$ cylinder is equal to an isolated one $F_{t}^{0}$, which is the expected behaviour of the model.

Accordingly, the critical angle $\alpha_{c}$ is defined as:

$$
\alpha_{c}=\left\{\begin{array}{cl}
\pi / 2 & \text { if } \hat{w}>2 \tilde{W}, \\
\arcsin \left(\frac{\hat{w}}{\tilde{W}}\right) & \text { otherwise } .
\end{array}\right.
$$

The first line in Eq. 11 represents the case where interactions between cylinders are occurring for any angle of attack $\alpha_{\infty}$ since the influencing region always overlaps downstream cylinders.

The focus in now made on the expression of the common ratio $q$. To assess this quantity, it is hypothesized that when cylinders interact, $q$ obeys to a linear relation with respect to the angle of attack $\alpha_{\infty}$. Otherwise, a constant value of is specified. Therefore,

$$
q=\left\{\begin{array}{cc}
1 & \text { if } \alpha_{\infty}>\alpha_{c}, \\
\left(\frac{1-q_{p}}{\alpha_{c}}\right) \alpha_{\infty}+q_{p} & \text { otherwise. }
\end{array}\right.
$$

with $q_{p}$ being the common term when the incoming flow is purely parallel to the tangential direction.

All the previous steps are summarized in the following pseudo Algorithm 1 used to compute the tangential force $F_{t}$ from flow and geometry inputs: 


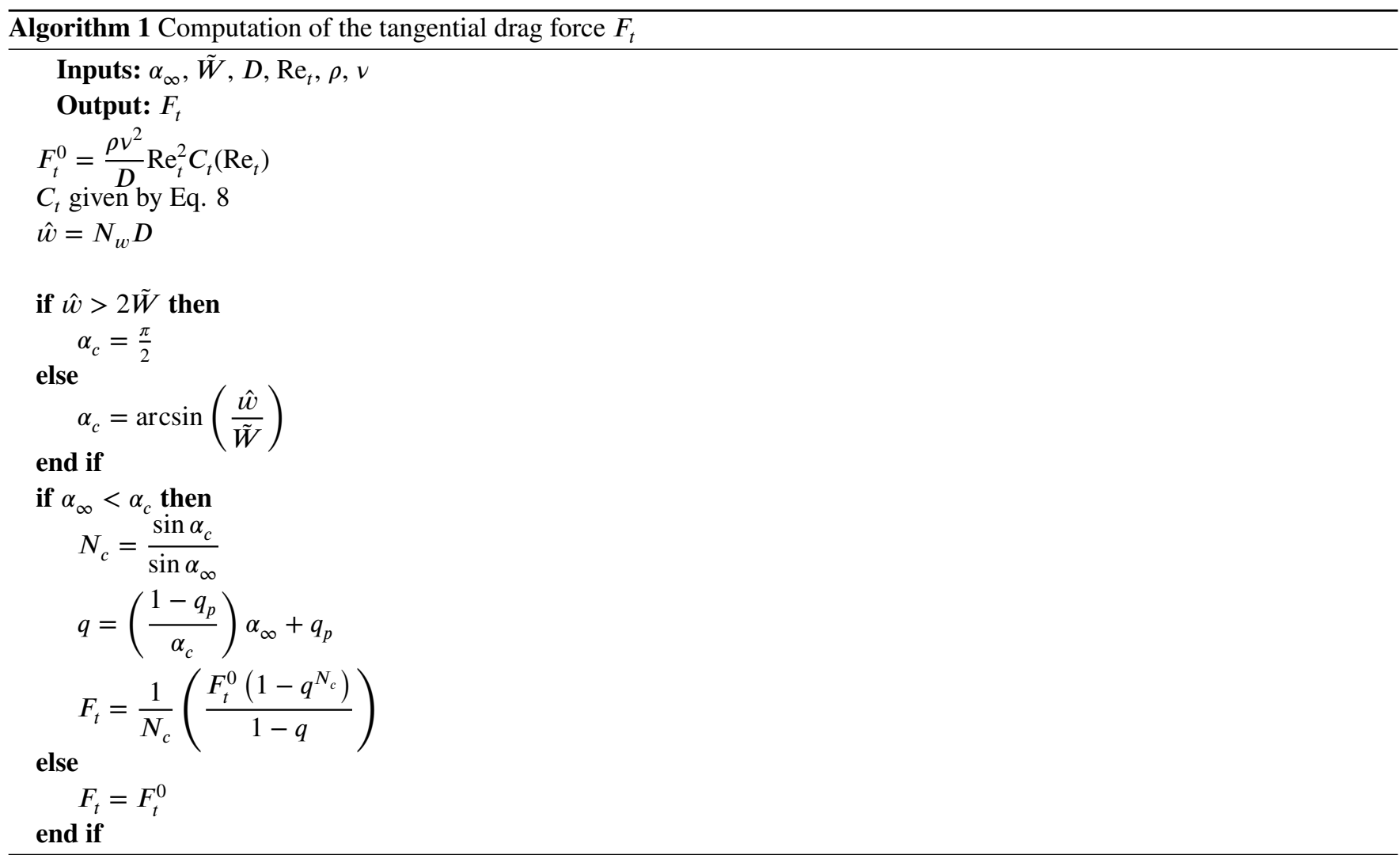

It should be noted that the division by zero induced by $q=1$ in Eq. 9 when cylinders do not interact is circumvented in Algorithm 1 by replacing Eq. 9 by $F_{t}^{0}$ directly.

For the tangential model to be fully complete, there remain two unknowns in the previous expressions: the width of the interaction area depending on $N_{w}$ and the common ratio $q_{p}$. Values for these parameters are determined by optimizing the agreement between the modelled tangential drag force given by Algorithm 1 with numerical values obtained from conformal CFD computations. Despite being built using 2-dimensional reasonings, it has been decided to perform 3D computations to calibrate the presented tangential model so as to partly account for the complex intra and extra-familial interactions between cylinders. Numerical details as well as boundary conditions of these 3D computations are fully described in Section 3.1 and not repeated here for the sake of brevity. Details regarding the fitting procedure with these 3D computations as well as the values for $N_{w}$ and $q_{p}$ are fully described in Appendix B to ease the reading.

\subsubsection{Longitudinal term $F_{l}$}

Though it is not depicted in Fig. 5, the longitudinal direction also experiences drag force from the fluid flow. Due to translational invariance in the longitudinal direction, it is hypothesized that cylinders are infinite and do not interact with each other longitudinally. Consequently, $F_{l}$ is independent of the $\tilde{W} / D$ ratio and only depends on the total Reynolds number Re a priori. This amounts to considering an isolated infinite cylinder subjected to an angle-oriented flow, a situation which has already been studied by Marheineke and Wegener ${ }^{[45]}$. Note that the longitudinal direction I notation in the present work corresponds to the tangential one $\tau$ in $[45$.

As opposed to the previous normal $F_{n}$ term, the $F_{l}$ does not only depend on the longitudinal Reynolds number $\operatorname{Re}_{l}$. More specifically, Marheineke and Wegener ${ }^{[45]}$ formulate that:

$$
F_{l}(\operatorname{Re})=F_{l}\left(\operatorname{Re}_{n}, \operatorname{Re}_{l}\right)=\frac{\rho v^{2}}{D} \operatorname{Re}_{n} \operatorname{Re}_{l} C_{l}\left(\operatorname{Re}_{n}\right)
$$


with $C_{l}$ being the dimensionless drag coefficient in the longitudinal direction. It should be noted that it depends only on the normal Reynolds number $\operatorname{Re}_{n}$ and is given by

$$
C_{l}\left(\operatorname{Re}_{n}\right)=\exp \left[\sum_{j=0}^{3}\left(p_{l, j}\left(\ln \operatorname{Re}_{n}\right)^{j}\right)\right]
$$

with $p_{l, 0}=1.1552, p_{l, 1}=-6.8479 \times 10^{-1}, p_{l, 2}=1.4884 \times 10^{-2}$ and $p_{l, 3}=7.4966 \times 10^{-4}$. Eq. 14 is valid in the interval $\operatorname{Re}_{n}=[0.1,100]$, which encompasses the intended range of interest relevant to the proposed heterogeneous model.

\section{Summary}

For each wire of the endovascular device, the normal, tangential and longitudinal components are computed thanks to Eq. 4 Algorithm 1 and Eq. 13, respectively. These formulae are applied for each family of wires (blue and red in Fig. 4) and then added up. As a note of caution, it should be mentioned that the total force $\mathbf{F}_{\mathbf{m}}$ given by Eq. 2 stands for drag force experienced by each wire. Due to the reciprocity principle, $-\mathbf{F}_{\mathbf{m}}$ is regularized by the IBM procedure to mimic the force applied by the struts onto the fluid.

\section{2 | Edge-based IBM regularization}

Now that the linear force relevant to each wire segment is known, it must be regularized onto the fluid mesh, as illustrated in Fig. 6 It should be noted that for the proposed model to be applicable, the solid part must have 2 dimensions less than the fluid, i.e. 1D struts for 3D fluid. Nevertheless, and for the sake of simplicity and explanations, Fig. 6/represents 1D wires immersed in a $2 \mathrm{D}$ view, which can be seen as a slice through a $3 \mathrm{D}$ domain, passing through device wires.

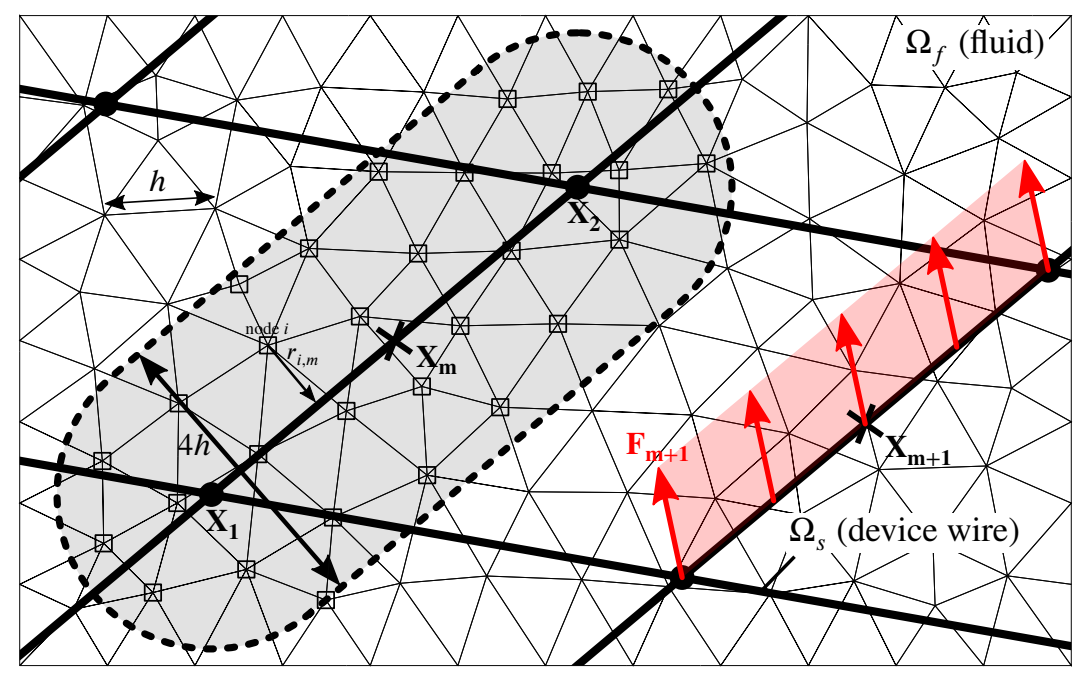

FIGURE 6 Edge-based IBM main components and notations. The support of the modified window function $\bar{w}\left(r_{i, m}\right)$ Eq. 16 is depicted in grey. It has an ovoid shape around the $\mathrm{m}^{\text {th }}$ edge. The square points represents the fluid nodes which are affected by the constant linear density of force $\mathbf{F}_{\mathbf{m}}$ exerted by the $\mathrm{m}^{\text {th }}$ edge.

The underlying notations and principles of the proposed edge-based IBM are now introduced. Solid and fluid domains are denoted by $\Omega_{s}$ and $\Omega_{f}$ respectively. In Fig. 6 , they correspond to the 1D solid wires (thick black lines) and the fluid mesh (thin black triangles) respectively. Capital letters are relevant to the solid domain while lower case denote fluid quantities. The force $\mathbf{f}_{\mathbf{i}}$ at the $\mathrm{i}^{\text {th }}$ fluid node is given by:

$$
\mathbf{f}_{\mathbf{i}}=\sum_{m=1}^{M} \mathbf{F}_{\mathbf{m}} \bar{w}\left(r_{i, m}\right),
$$


with $M$ the total number of wire segments in the neighbourhood of the $\mathrm{i}^{\text {th }}$ node and $\mathbf{F}_{\mathbf{m}}$ the linear density of force at the $\mathrm{m}^{\text {th }}$ solid edge (see the red vectors in Fig. 6. In the following, it will be assumed that this density is constant for each edge, reflecting the fact that the wire diameter $D$ is smaller than the inter-wire distance (hypothesis H2 described in subsubsection 2.1.1). In Eq. 15 , the $\bar{w}$ coefficients are collectively referred to as the modified regularization window; the way they are computed will be detailed in the remaining of this section. The edge-based methodology developed to compute the $\bar{w}$ coefficients builds heavily on the work of Pinelli et al. [46] and the RKPM principle introduced by Liu et al. ${ }^{477}$. Following the notations used by Sigüenza et al. $\stackrel{48 \mid}{ }$ and for a second-order RKPM, the coefficients $\bar{w}$ in Eq. 15 write:

$$
\begin{aligned}
& \bar{w}\left(r_{i, m}\right)=w\left(r_{i, m}\right) \times\left[\beta_{0}+\beta_{1}\left(\frac{x_{i}-X_{m}}{h}\right)+\beta_{2}\left(\frac{y_{i}-Y_{m}}{h}\right)+\beta_{3}\left(\frac{z_{i}-Z_{m}}{h}\right)\right. \\
& +\beta_{4}\left(\frac{x_{i}-X_{m}}{h}\right)\left(\frac{y_{i}-Y_{m}}{h}\right)+\beta_{5}\left(\frac{x_{i}-X_{m}}{h}\right)\left(\frac{z_{i}-Z_{m}}{h}\right)+\beta_{6}\left(\frac{y_{i}-Y_{m}}{h}\right)\left(\frac{z_{i}-Z_{m}}{h}\right) \\
& \left.+\beta_{7}\left(\frac{x_{i}-X_{m}}{h}\right)^{2}+\beta_{8}\left(\frac{y_{i}-Y_{m}}{h}\right)^{2}+\beta_{9}\left(\frac{z_{i}-Z_{m}}{h}\right)^{2}\right],
\end{aligned}
$$

with $\mathbf{x}_{\mathbf{i}}=\left(x_{i}, y_{i}, z_{i}\right)$ and $\mathbf{X}_{\mathbf{m}}=\left(X_{m}, Y_{m}, Z_{m}\right)$ the coordinates of the $\mathrm{i}^{\text {th }}$ fluid node and the middle of the $\mathrm{m}^{\text {th }}$ solid edge, respectively. $r_{i, m}$ stands for the distance between the $\mathrm{i}^{\text {th }}$ fluid node and the point projected onto the $\mathrm{m}^{\text {th }}$ solid edge, as depicted in Fig. 6 The original window function $w\left(r_{i, m}\right)$ appearing in Eq. 16 is written as:

$$
w\left(r_{i, m}\right)= \begin{cases}1+\cos \left(\frac{\pi r_{i, m}}{2 h}\right) & \text { if } r_{i, m}<2 h, \\ 0 & \text { otherwise, }\end{cases}
$$

with $h$ the characteristic length scale of the fluid mesh (see Fig. 6. The expression for $w\left(r_{i, m}\right)$ ensures that the volume source term added to the Navier-Stokes equations is zero everywhere except in the vicinity of solid edges, i.e. inside the $4 h$ ovoid grey region in Fig. 6 In other words, it enables to restrict the extent to which the fluid can feel the solid parts and increases as approaching the wires. The $4 h$ width of the window function support for edge-based IBM is a classical value in node-based $\mathrm{IBM}^{[30 \mid 48}$, but it is actually a free parameter. More precisely, it has been found in our tests that a width of $6 h$ offers a good compromise between errors and numerical stability while properly representing on the fluid mesh the jetting-flow taking place between consecutive struts.

The RKPM coefficients $\beta_{k}$ in Eq. 16 stand for the 10 unknowns coefficients of the correction polynomial which are calculated to ensure conservation of solid quantities when being regularized onto the fluid. Specific details on how these coefficients are obtained for each edge can be found in Appendix A.

\section{3 | VALIDATIONS}

In order to validate the heterogeneous model and its implementation, two sets of numerical simulations have been performed: idealized and patient-specific. In the following, results labelled by 'edge-based IBM' will refer to the proposed model. They will be compared to conformal computations which we refer to as the gold standard. All conformal and IBM simulations are performed with the same fluid properties and numerical discretization schemes. Broadwell Intel Xeon E5-2690V4 2,60 GHz cores with $128 \mathrm{~GB}$ of random-access memory were used for all computations.

\subsection{Idealized device geometry}

\section{Numerical setup}

An idealized configuration is first considered: an infinite planar device consisting of two sets of parallel straight wires intersecting at an inner angle $\alpha=\pi / 2$ is subjected to an incident velocity $\mathbf{U}_{\infty}$. $\mathbf{U}_{\infty}$ is varied, as well as the spacing between the struts, with the constraint that $\tilde{W} / D=W / D \gg 1$. To compute such a flow, a reduced computational domain is used (see Fig. 7), with periodic boundary conditions enforced at the left-right and front-bottom faces. The size of the domain is detailed in Fig. 7 . At the inlet, the uniform velocity field $\mathbf{U}_{\infty}$ is applied (see the red section in Fig. 7). The outlet section (black section in Fig. 7) was 
placed further downstream to prevent any interactions with the wakes of the wires. A convective outflow condition was applied at the outlet.

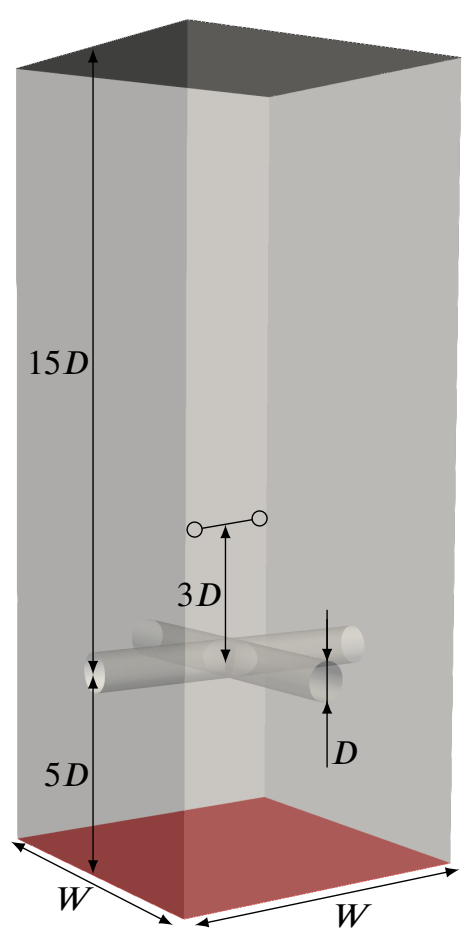

FIGURE 7 3D bi-periodic domain of width $W$. No-slip boundary conditions are applied on the struts surfaces. The angle between the wires is $\pi / 2$. Inlet and outlet sections are highlighted in red and black, respectively. Periodic boundary conditions are enforced at the left-right and front-bottom faces. The probing line which was used to compute the error defined by Eq. 18 is also depicted downstream of the cylinders (line with o symbols).

Compared to the 2D configuration depicted in Fig. 5 the imposed unperturbed velocity is now free to be outside the (n,t) plane. The definition of $\alpha_{\infty}$ in the $(\mathbf{n}, \mathbf{t})$ plane still holds and is now denoted by $\alpha_{\infty}^{n, t}$; the second angle necessary to define the orientation of the unperturbed velocity is denoted by $\alpha_{\infty}^{l, t}$ and lies in the $(\mathbf{l}, \mathbf{t})$ plane. In the following, two values of $\alpha_{\infty}^{l, t}$ have been considered: 0 and $\pi / 3$, the latter being chosen to study the situation where the flow is not aligned with any of the wires, which is the general case in real flow conditions. The following ranges have been considered for the other varying parameters: $W / D \in[6,20], \alpha_{\infty}^{n, t} \in[\pi / 20, \pi / 2]$ and $\operatorname{Re}=\frac{\left\|\mathbf{U}_{\infty}\right\| D}{v} \in[2.5,20]$. Computations at $\operatorname{Re}=2.5$ were performed so as to assess the behaviour of the model when being used outside its working range, i.e. at low Reynolds numbers. In other words, this flow condition cannot be used to validate the model. For each operating point defined by values of $\left(\operatorname{Re}, \frac{W}{D}, \alpha_{\infty}^{n, t}, \alpha_{\infty}^{l, t}\right)$, a conformal and an edge-based IBM simulation were performed, resulting in 580 numerical computations in total. For all conformal computations, no-slip boundary conditions were applied at the strut surface.

Both conformal and IBM meshes have been built using $\mathrm{Gmsh}^{[49]}$. A smooth mesh size coarsening when moving away from cylinders locations has been specified. In the conformal domains, at the cylinders surface, a mesh size $h$ was enforced to be a function of the perimeter such that $h=\frac{\pi D}{N_{h}^{c}}$ with $N_{h}^{c}=40$. For edge-based IBM grids, the mesh size $h$ was given as a function of the inter-wire distance $W$ such that $h=\frac{W}{N_{h}^{\mathrm{IBM}}}$ with $N_{h}^{\mathrm{IBM}}=20$. Several IBM computations have also been performed for $N_{h}^{\mathrm{IBM}}=10$ to demonstrate the independence of the results with respect to the grid discretization. The larger conformal mesh that has been built for $W / D=20$ computations consists of $19 \mathrm{M}$ tetrahedral elements in total.

Tab. 1 summarizes the 3D simulation parameters used to validate the proposed model. It should be noted that this validation step aims at verifying that the whole heterogeneous methodology, i.e. the drag models and the edge-based IBM, is producing realistic results. In this view, the undisturbed velocity $\mathbf{U}_{\infty}$ is artificially given as an input to the drag models since it is fully known at the inlet section. 


\begin{tabular}{lccccc}
\hline & $\operatorname{Re}$ & $W / D$ & $\alpha_{\infty}^{n, t}$ & $\alpha_{\infty}^{l, t}$ & Mesh size $h$ \\
\hline Conformal & $\left\{2.5^{*}, 5,10,15,20\right\}$ & $\{6,8,10,20\}$ & $\frac{\pi}{20} \rightarrow \frac{\pi}{2}$ & $\left\{0, \frac{\pi}{3}\right\}$ & $\frac{\pi D}{40}$ \\
Edge-based IBM & & & $\left\{\frac{W}{20}, \frac{W}{10}\right\}$ \\
\hline
\end{tabular}

TABLE $13 D$ conformal and edge-based IBM computation parameters.

Since YALES2BIO solves the unsteady Navier-Stokes equations, temporal convergence of the simulations was assessed by measuring the forces integrated over the cylinders' walls for conformal simulations, thus enabling to obtain a physical steady time for each operating point. Edge-based IBM computations were ran until the conformal physical steady time was reached.

Conformal and IBM results were compared qualitatively using velocity magnitude contours on specific slices. Quantitative errors were also computed on downstream velocity profiles. More precisely, a probing line of length $L$ diagonal to the numerical domain and located three diameters downstream of the device was used to get velocity profiles for both IBM and conformal results (see Fig. 7). Then, the relative error $E$ in $\%$ was computed as:

$$
E=100 \times \frac{1}{L} \int_{0}^{L} \frac{\left\|\mathbf{U}_{\mathrm{IBM}}-\mathbf{U}_{\mathbf{c}}\right\|}{\left\|\mathbf{U}_{\infty}\right\|} \mathrm{d} l,
$$

with $\mathbf{U}_{\mathrm{IBM}}$ and $\mathbf{U}_{\mathbf{c}}$ being the IBM and conformal velocity vectors along the probing line, respectively.

All post-processing steps were performed using the Visualtization Toolkit (VTK) library [50] and Paraview [51] software. Each parallel computation has been performed using one processing unit every approximately 100,000 tetrahedra, which yields a good balance between communication and computation costs for the current applications. The number of processors ranged from 28 to 196, the latter being reached for $W / D=20$ meshes.

\section{Results}

Fig. 8 gives a qualitative insight into velocity fields on a slice placed diagonally to the 3D domain for both conformal and IBM modalities when the angle $\alpha_{\infty}^{n, t}$ decreases, for the typical operating point $\left(\operatorname{Re}, \tilde{W} / D, \alpha_{\infty}^{l, t}\right)=(5,8,0)$. Very good trends are obtained with the heterogeneous model, whatever the inclination angle of the incident velocity. However, it appears that edgebased IBM downstream velocities are systematically underestimated compared to conformal ones when $\alpha_{\infty}^{n, t}>\pi / 2$, which is particularly visible when the incoming velocity is close to being purely tangential. Conversely, it is not the case when $\alpha_{\infty}^{n, t}=$ $\pi / 2$ since the recirculation region appears to be longer for conformal computations. These trends have also been found for other (Re, $\left.\tilde{W} / D, \alpha_{\infty}^{l, t}\right)$ operating points (not showed here). This suggests that for non-normal incoming flow situations, the drag forces applied on the fluid are too high and overestimate the incoming flow blockage. This overestimation increases with the tangential component of the incoming flow. Nevertheless, the current model correctly reproduces relevant velocity patterns such as redirection due to the cylinders' presence, downstream wakes and jetting-flow between the wires compared to conformal fields, using grid sizes coarser by a factor of 6 approximately.

Quantitative comparisons between IBM and conformal approaches can be found in Fig. 9 for all the parameters ranges given in Tab. 1 For each value of $\alpha_{\infty}^{n, t}$, the solid line represents the mean error and the opaque filling goes from the minimum to the maximum error for all the $\tilde{W} / D$ ratios studied.

As expected, the computations performed outside the intended range of applicability of the model, i.e. at a diffusive flow regime where $\operatorname{Re}=2.5$, exhibit very high errors, reaching almost $140 \%$ for tangential inflow conditions. As already stated, these computations were performed to demonstrate that the model cannot be used for diffusive regimes. Therefore, the results presented in the following section only focus on computations performed for $\operatorname{Re}>2.5$.

The overall agreement is very good: errors above $25 \%$ were attained by only $11 \%$ of all computations. Among all the operating points, the maximum error reached $59 \%$ and was obtained for $\left(\operatorname{Re}, \tilde{W} / D, \alpha_{\infty}^{n, t}, \alpha_{\infty}^{l, t}\right)=(5,6, \pi / 20, \pi / 3)$. Studying each Re individually, it appears that the mean and min-max errors are increasing with decreasing $\alpha_{\infty}^{n, t}$ for $\operatorname{Re} \in[5,10]$, which is the opposite for $\operatorname{Re}=20$. For $\operatorname{Re}=15$, the errors seem to be constant for all geometric and flow conditions.

Despite not being depicted in Fig. 9, it has been noticed that the maximum errors are obtained for low $\tilde{W} / D$ and low $\alpha_{\infty}^{n, t}$ values, i.e. when cylinders are close to each others and when the incoming flow is close to being purely tangential, which implies strong interactions between cylinders. This indicates that both the tangential and longitudinal components of the force model, 


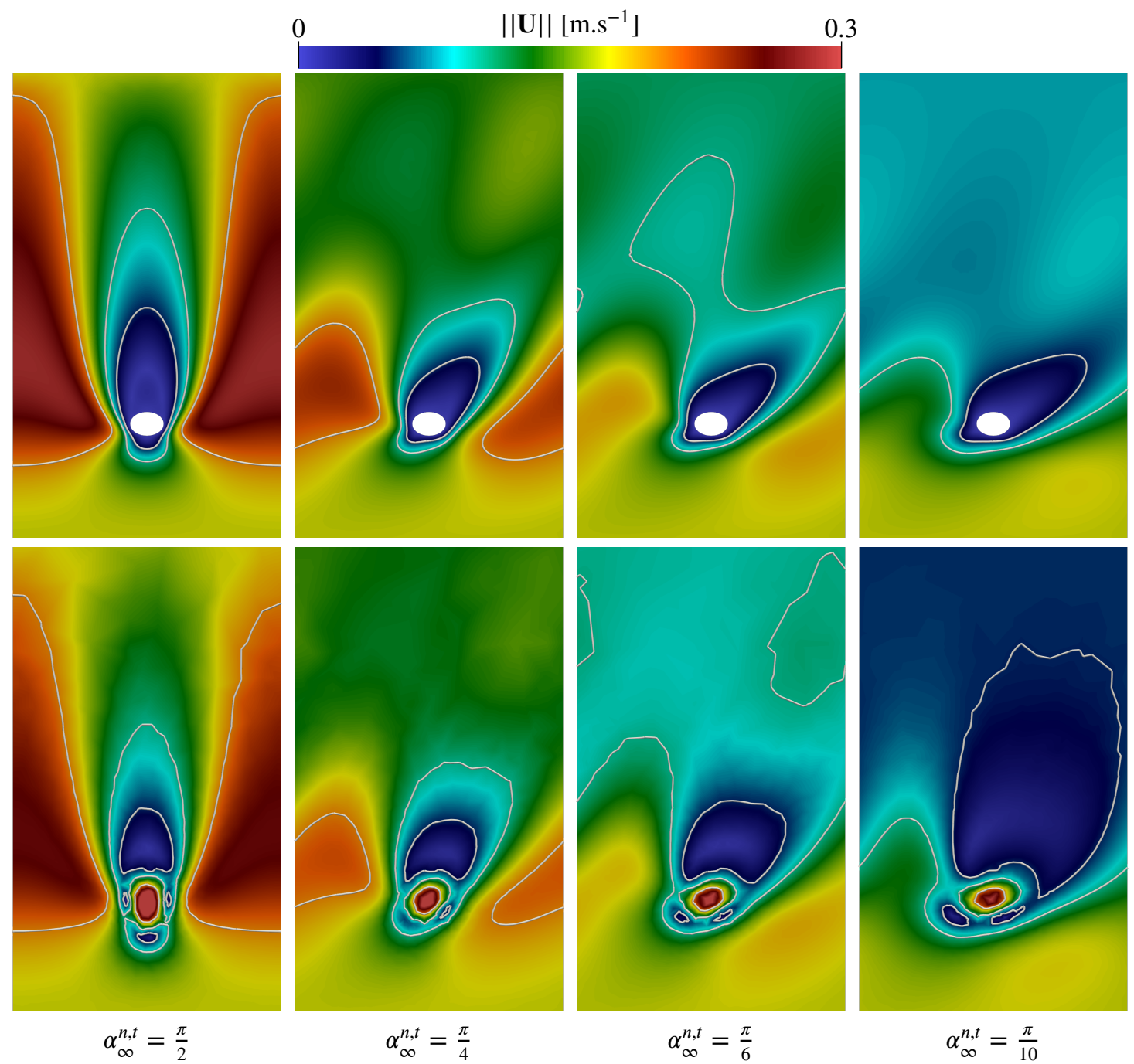

FIGURE 8 Velocity fields and contours for conformal (first line) and edge-based IBM (second line) approaches on a plane placed diagonally to the $3 \mathrm{D}$ domain for various $\alpha_{\infty}^{n, t}$ angle of attack. The chosen operating point $\left(\operatorname{Re}, \tilde{W} / D, \alpha_{\infty}^{l, t}\right)$ is equal to $(5,8,0)$. A good qualitative agreement is reached by the current model when being compared to conformal velocity fields.

which are higher than the normal one for these situations, do not capture sufficiently well intra-familial interactions between cylinders and that additional mechanisms such as extra-familial interactions and crossing effects, which have not been modelled in this work, might be at stake and important to take into account.

The 'real-flow condition' where the incident flow is not aligned with the cylinders $\left(\alpha_{\infty}^{l, t}=\pi / 3\right)$ yield similar results as the case $\left(\alpha_{\infty}^{l, t}=0\right)$, albeit with a small increase in the mean and min-max errors. Nevertheless, these errors were considered to be acceptable, thus demonstrating the robustness of the current model when being used in any incoming flow-conditions.

No significant differences on the obtained errors were noticed when increasing the mesh size by a factor of two (green line in Fig. 9p. 

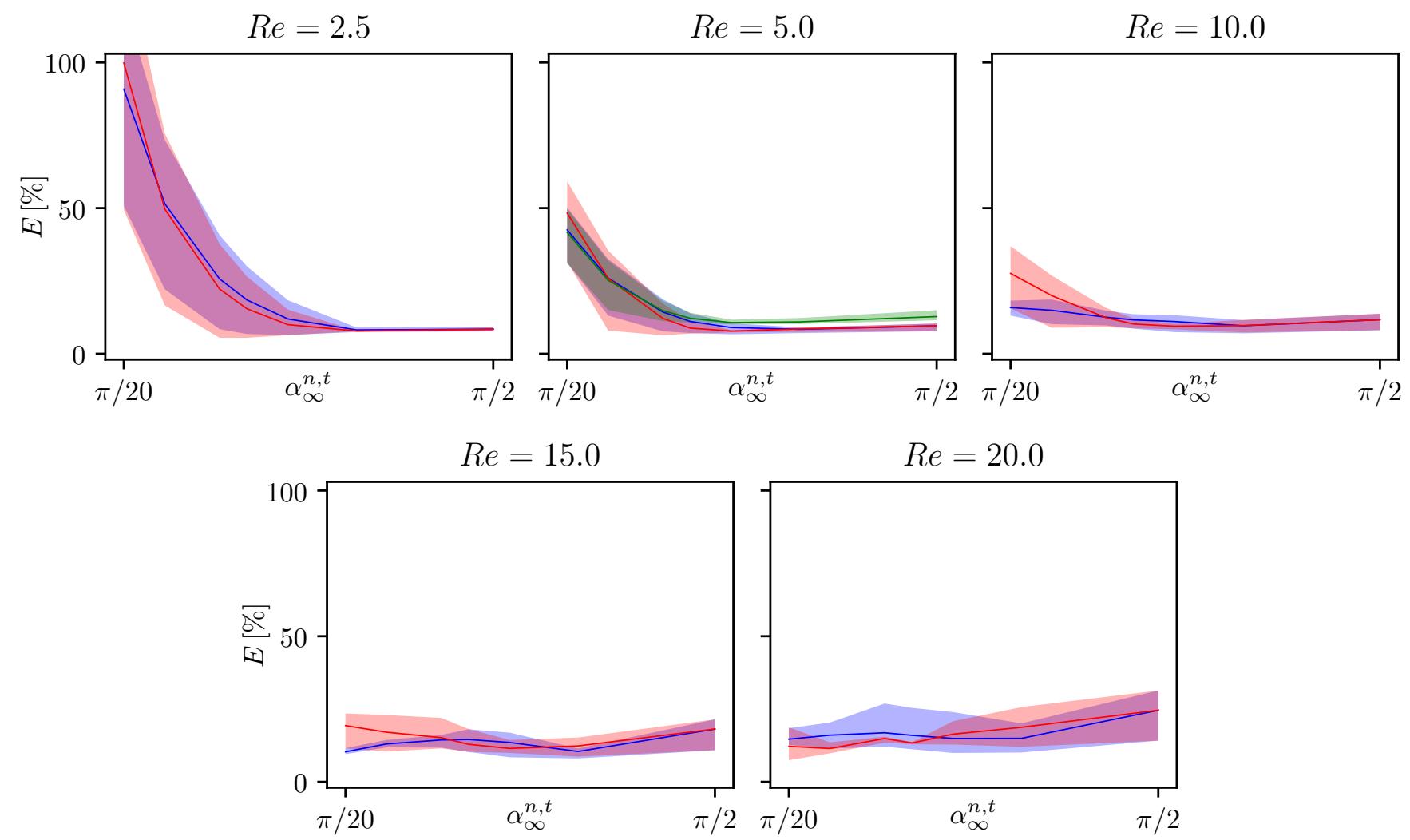

FIGURE 9 Errors given by Eq. 18 for $\alpha_{\infty}^{l, t}=0(-)$ and $\alpha_{\infty}^{l, t}=\pi / 3(-)$ at $h=\frac{W}{20}$ and $\alpha_{\infty}^{l, t}=0(-)$ at $h=\frac{W}{10}$. The solid line and the opaque filling represent the mean and min-max errors for all the $\tilde{W} / D$ ratios at a given angle $\alpha_{\infty}^{n, t}$ respectively. 


\section{2 | Patient-specific device geometry}

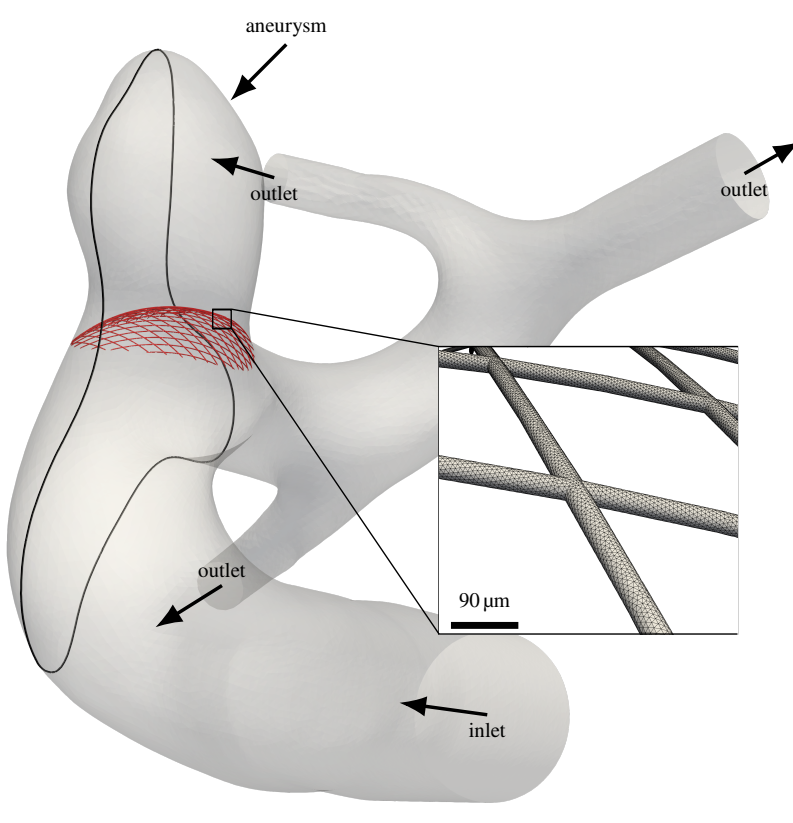

(a)

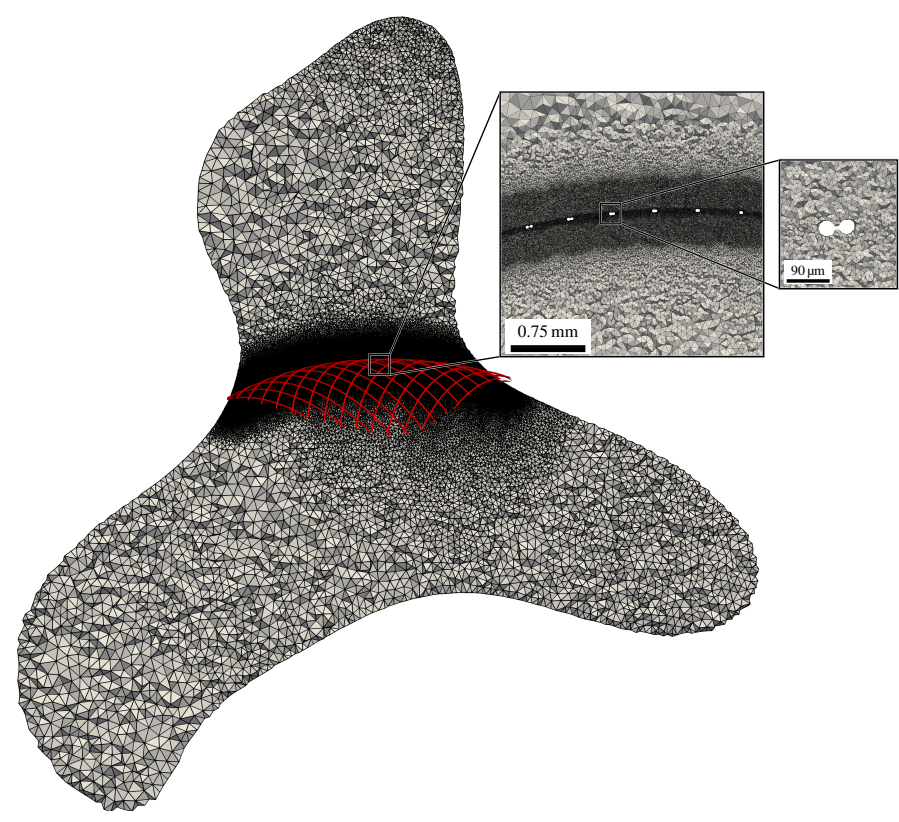

(b)

FIGURE 10 Insights into geometry and meshing characteristics of the aneurysm case. a: 3D patient-specific arterial geometry harbouring an intracranial aneurysm treated by a flow-diverter (in red). Note that distal and proximal portions of the device in apposition with the parent artery have been removed. The close-up view shows the surface discretization of the struts. The black line on the arterial surface represents the intersection with the plane used for further analysis. Inlet and outlet sections are denoted with black arrows. b: Previously defined plane showing the volume mesh sizes at the aneurysm sac, the parent artery and the neck. Two successive zooms enable to appreciate the smooth mesh size coarsening when moving away from the wires (small white holes).

\section{Numerical setup}

To illustrate the capabilities of the proposed model, the hemodynamics inside the aneurysm of a patient treated by a commercial flow-diverter was studied. Starting from medical images, the arterial surface has been reconstructed using the Marching Cubes algorithm 52 . As depicted in Fig. 10 the arterial geometry consists of a saccular aneurysm (black arrow) located at the ICA (Internal Carotid Artery) portion of the vasculature. The parent artery extends up to two subsequent arterial bifurcations. The surface went through multiple pre-processing steps using the VMTK (Vascular Modelling Tool Kit) library [53]. Smoothing and addition of flow extensions at each opening have been performed. Surface remeshing using distance to centerlines as a meshing size constraint has been carried out. This provides a constant number of elements across all arterial diameters.

Then, the endovascular device was numerically deployed into the previously pre-processed surface. The length and the diameter of the device were chosen in order to start and end into straight arterial sections without occluding the downstream arterial bifurcation. The device consists in 48 wires of $30 \mu \mathrm{m}$ in diameter with an inter-wire distance $W=0.335 \mathrm{~mm}$, thus giving a ratio $W / D \approx 11$. Numerical mechanical deployment was performed with the use of beams elements for each wire of the device. The arterial surface was considered to be rigid and static. The LS-DYNA solver http://www.lstc.com/products/ls-dyna was used to solve the mechanical deployment and handle the contact between the device and the arterial wall.

Three sets of meshes were then built for each of the CFD computations: device-free, conformal and edged-based IBM. The device-free volume mesh inherited grid sizes from the previously pre-processed surface. The grid for the IBM simulations has been obtained by refining the surface and volume of the device-free grid in order to ensure a mesh size $h=w / 8$ in the vicinity of the wires. They consist of $3 \mathrm{M}$ and $6.5 \mathrm{M}$ tetrahedra, respectively. 
As opposed to the previous ones, generating the conformal mesh required more steps. First, distal and proximal portions of the device in contact with the arterial walls were removed to save computational costs. Thus only the part immersed in the neck region has been conserved, has depicted by Fig. 10 Then, circular disks have been extruded along the neutral axis of the wires outputted by LS-DYNA and boolean unions were applied to link all the wires as one surface. This step has been performed using both Gmsh and VTK. The device's surface has been discretized with a mesh size $h=\frac{\pi D}{15}$, which is almost an order of magnitude finer that commonly applied in the literature ${ }^{[14}$. Next, the device surface was removed from the computational domain using the boolean tool available in the Blender software (https://www.blender.org/). Then, surface remeshing and cleaning was performed using VMTK and MeshLab (http://www.meshlab.net/), respectively. Finally, a first volume mesh was generated with VMTK and then refined near the struts with MMG3D ${ }^{[54}$ to provide a smooth transition from fine to coarse regions of the mesh, as depicted by Fig. 10. It should be noted that a constant mesh size has been specified in a layer around the wires to better capture cylinder's wakes. The final mesh consists of $120 \mathrm{M}$ tetrahedra.

To reduce the computational burden, only the stationary case was considered when analysing the performance of the heterogeneous model with respect to the conformal case. From a physical point of view, this amounts to considering that the perturbations induced by the network of wires adapt instantly when the outer flow conditions change. This is indeed well justified since the typical time scale of the pulsatile flow rate signal through the parent artery (of order $0.1 \mathrm{~s}$, say) is very large compared to the typical time scale of the flow around each wire $\left(2 \times 10^{-4} \mathrm{~s}\right.$ and $5 \times 10^{-5} \mathrm{~s}$ for the diffusion and convection times, respectively).

At the inlet, a fully-developed Poiseuille velocity profile has been specified. Peak systolic flow regime was used to simulate a 'worst-case scenario', since the maximum velocities are observed during this phase. First, a mean flow rate has been calculated using the ICA diameter as recommended by Valen-Sendstad et al. ${ }^{[55]}$ and Chnafa et al. ${ }^{[56]}$. Then, it has been plugged onto the generalized flow waveform measured on older adults given by Hoi et al. [57], which resulted in a peak-systolic flow rate of $418.8 \mathrm{ml} . \mathrm{min}^{-1}$. The inlet Reynolds number was equal to $\operatorname{Re}=\frac{U D}{v}=594$, based on the inlet diameter.

As classically done in hemodynamic simulations of the flow in cerebral aneurysms, blood was assumed to be an incompressible Newtonian fluid with kinematic viscosity $v=3.5 \times 10^{-6} \mathrm{~m}^{2} \cdot \mathrm{s}^{-1}$ and density $\rho=1.06 \times 10^{3} \mathrm{~kg} \cdot \mathrm{m}^{-3}$ [58]. Arterial walls (and device's surface for the conformal mesh) were assumed to be rigid with a no-slip boundary condition. At the outlets, a convective outflow condition was specified such that

$$
\frac{\partial \mathbf{u}}{\partial t}+U_{\text {conv }} \frac{\partial \mathbf{u}}{\partial \mathbf{n}}=0
$$

with $\mathbf{n}$ the outward normal to the outlet surfaces, and $U_{\text {conv }}$ the convective velocity adjusted at each outlet. Its value was computed using the ratio of each outlet surface over the sum of the outlet surfaces, which ensures global mass conservation over the entire flow domain.

When dealing with complex geometries and incident flows as it is the case for this validation step, the concept of unperturbed upstream velocity $\mathbf{U}_{\infty}$ is not as clear as in the idealized validation Section 3.1. Indeed, the flow interacting with the device is not uniform and its structure is dictated by the shape of the parent artery as well as the corresponding time-dependent flow rate. The only option to ensure the operability of the model in this situation is to reconstruct $\mathbf{U}_{\infty}$ from local and instantaneous information available in the device region. Since mass conservation is at work in the close vicinity of the wires, it yields that the normal component of $\mathbf{U}_{\infty}$ can be obtained from the flow rate through the diamonds formed by the struts (see the left of Fig. 4). Reconstructing the other components of the unperturbed velocity $\mathbf{U}_{\infty}$ from local quantities is more challenging since there is no conservation principle at play. To achieve that, a 2D version of the 3D numerical computations presented in Section 3.1 was used to derive an empirical law linking the measured flow angle at the wires with the imposed $\alpha_{\infty}$ one.

Qualitative and quantitative comparisons between conformal and edge-based IBM results were performed using magnitude velocity contour on the slice depicted in Fig. 10 as well as by computing the mean velocity magnitude over the aneurysm sac defined as:

$$
\overline{U_{a}}=\frac{1}{V_{a}} \int_{\Omega_{a}}\|\mathbf{U}\| \mathrm{d} V
$$

with $\Omega_{a}$ the aneurysm sac domain of volume $V_{a}$ manually delineated on ParaView. $\overline{U_{a}}$ measures the intensity of the flow in the $\operatorname{sac}^{[14}$. Histograms of intra-saccular velocity magnitude were also used to compare both modalities, the conformal results being considered as the reference data. 


\section{Results}

Qualitative comparisons of blood velocities in the vicinity of the aneurysm neck and in the parent artery can be found in Fig. 11 which displays velocity magnitude contours and 3D-projected fields for no-device, edge-based IBM and conformal approaches. Compared to the no-device case, the conformal figure shows that the jet coming from the parent artery is reduced by the implanted device, yielding a decrease of intra-saccular velocities and a displacement of the center of the recirculating region in the sac. This trend is well captured by the edge-based IBM model, despite being less pronounced than in the reference results.

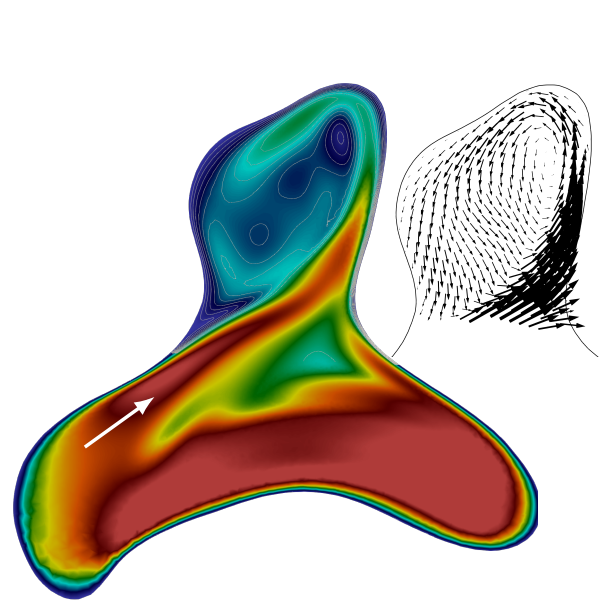

(a)

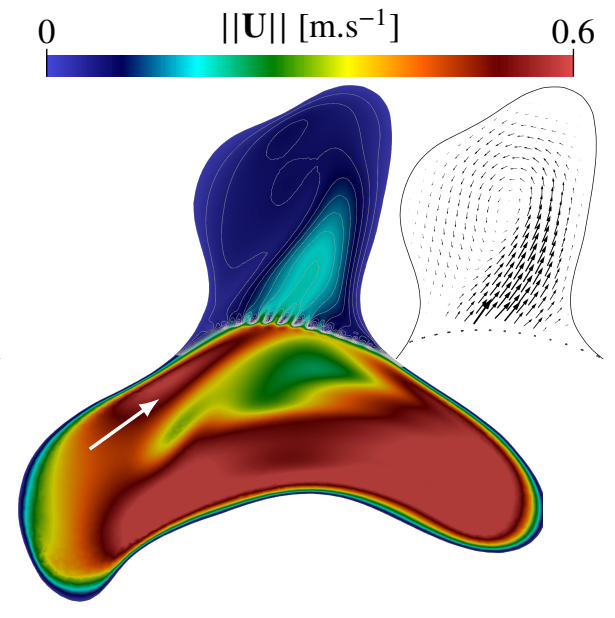

(b)

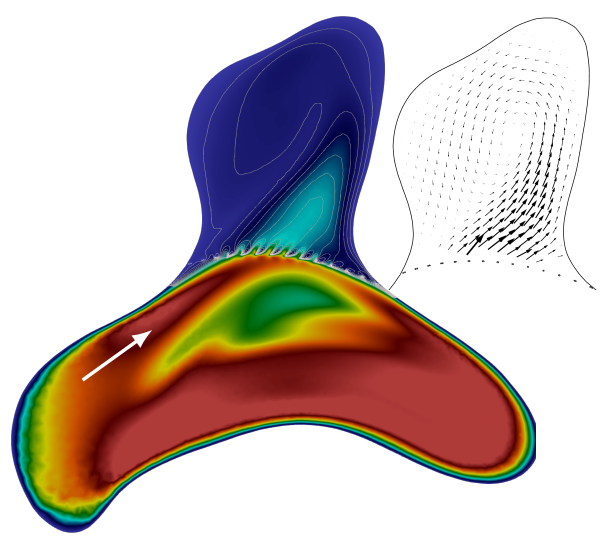

(c)

FIGURE 11 Velocity field slices for a: no device, b: edge-based IBM and c: conformal configurations. The white arrow shows the main direction of the flow. Velocity vectors are projected onto the slice to ease flow visualization.

Nevertheless, wakes and jetting-flows between the struts are comparable in the IBM and in the conformal simulations, which demonstrates the robustness of the model when being employed in a realistic geometry. It should be noted that the maximum Reynolds number Re reconstructed from the infinity velocity $\mathbf{U}_{\infty}$ reached 8 in this case, which is in the middle of the intended range of applicability of the proposed method.

To further study the qualitative performances of the model, volume rendering of vorticity magnitude $\|\mathbf{\Omega}\|=\|\boldsymbol{\nabla} \times \mathbf{U}\|$ is depicted in Fig. 12 It should be noted that velocity gradients due to boundary layers close to the arterial wall were automatically removed from the volume fields to enhance flow visualization. Due to the presence of very large vortices, the opacity transfer function was chosen to be a non-linear function (see the black line on the color bar).

As showed by Fig. 12, two pair of vortices are created in the parent artery, stemming from the upstream curvature in this region. These vortices do not appear to be impacted by the presence of the device. Nevertheless, the struts strongly reduce intra-saccular vorticity compared to the no-device configuration. This behaviour is correctly captured by the proposed model which exhibits velocity gradients due to the wires that are similar to the conformal approach. Downstream vortices in the second bifurcation appear to be impacted by the endovascular device, which is also correctly reproduced by the edge-based IBM method.

Quantitative comparisons between no-device, IBM and conformal modalities can be found in Fig. 13 which shows the probability density function (PDF) $\varphi$ and spatially averaged values of intra-saccular velocity magnitude. The device entails a significant decrease of the velocity magnitude and the two peaks PDF produced by the device is well retrieved by the edge-based model. When being compared to no-device, IBM and conformal induce a reduction of $\overline{U_{a}}$ by $58 \%$ and $66 \%$ respectively. Comparing $\overline{U_{a}}$ values, the proposed IBM model exhibits a relative error of $20 \%$ with respect to the conformal situation.

To enable quantitative comparisons of computational costs between IBM and conformal modalities, the memory used by all the processing units was gathered. Additionally, the two following reduced computational time measures were computed:

$$
\left\{\begin{array}{l}
\mathrm{RCT}_{1}=\frac{T_{w c} N_{p}}{N_{c} N_{i t}} \\
\mathrm{RCT}_{2}=T_{w c} N_{p}
\end{array}\right.
$$




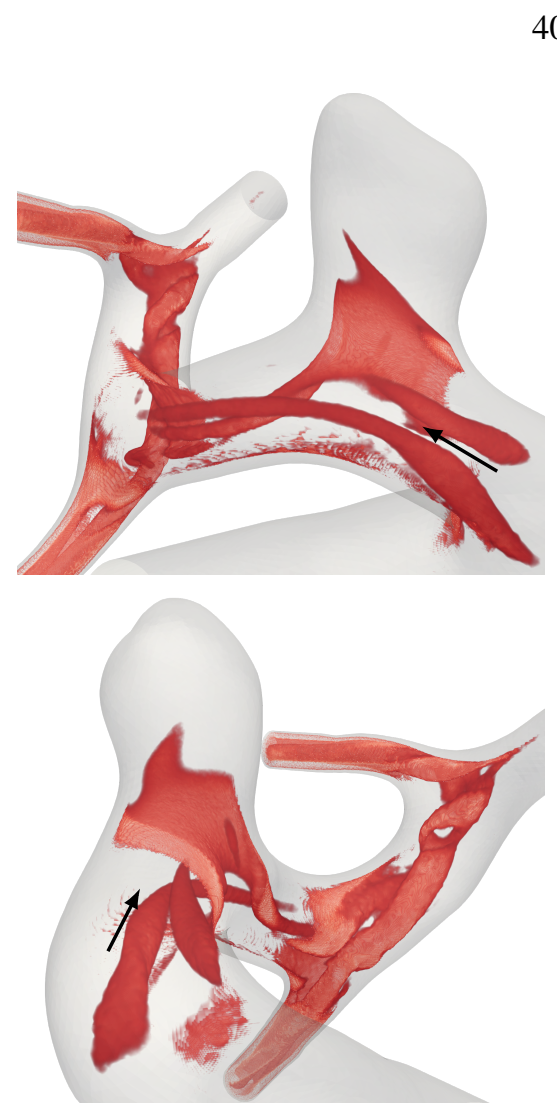

(a)
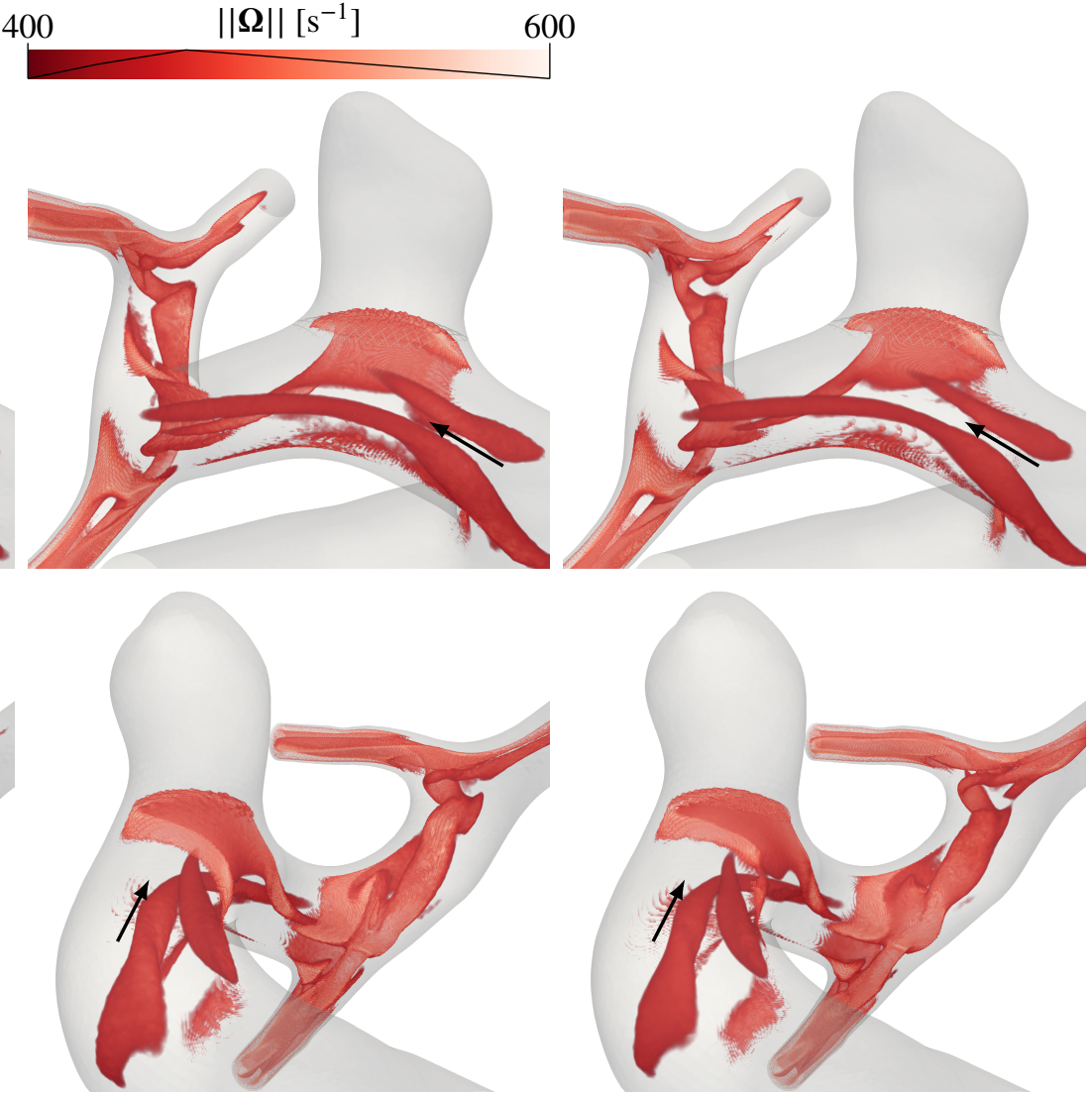

(b)

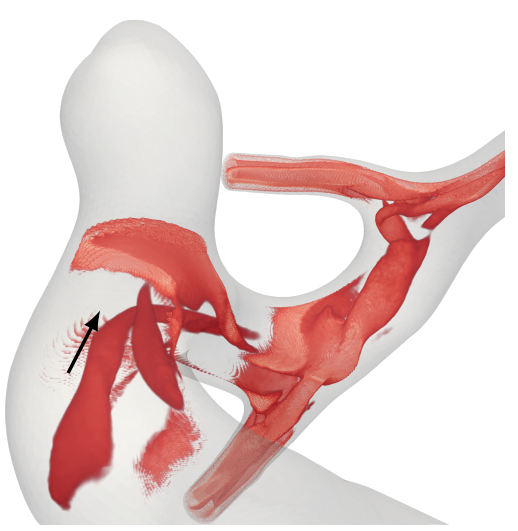

(c)

FIGURE 12 Volume rendering of vorticity magnitude $\|\boldsymbol{\Omega}\|=\|\boldsymbol{\nabla} \times \mathbf{U}\|$ for $\mathbf{a}$ : no-device, $\mathbf{b}$ : edge-based IBM and c: conformal configurations for two point of views. The arterial wall is made partially transparent and velocity gradients due to boundary layers at the wall were automatically removed to enhance flow visualization. The opacity transfer function is given directly over the color bar (black line). Black arrows show the main direction of the flow.

with $T_{w c}$ the wall-clock time, $N_{p}$ the number of processing units involved in the parallel computation, $N_{c}$ the total number of cells in the numerical domain and $N_{i t}$ the total number of iterations. $\mathrm{RCT}_{1}$ enables to study the supplementary cost induced by the proposed model compared to a no-model computation. $\mathrm{RCT}_{2}$ serves to compare two parallel computations when the number of processors, the mesh and the involved numerical models are different, which is the case here. More specifically, it corresponds to the wall-clock computational time needed by a single processor to reach the desired physical time of the simulation.

The costs summarized in Tab. 2 demonstrate a drastic reduction of both memory usage and $\mathrm{RCT}_{2}$ by factors of 22 and 5766 respectively. $\mathrm{RCT}_{2}$ reduction can be mainly explained by the time-stepping gain between IBM and conformal, which has been reduced by a factor 300 approximately. $\mathrm{RCT}_{1}$ indicates that the model increases the computational cost per iteration by $12 \%$, which is considered to be acceptable with regards to the $\mathrm{RCT}_{2}$ and memory usage reductions. 

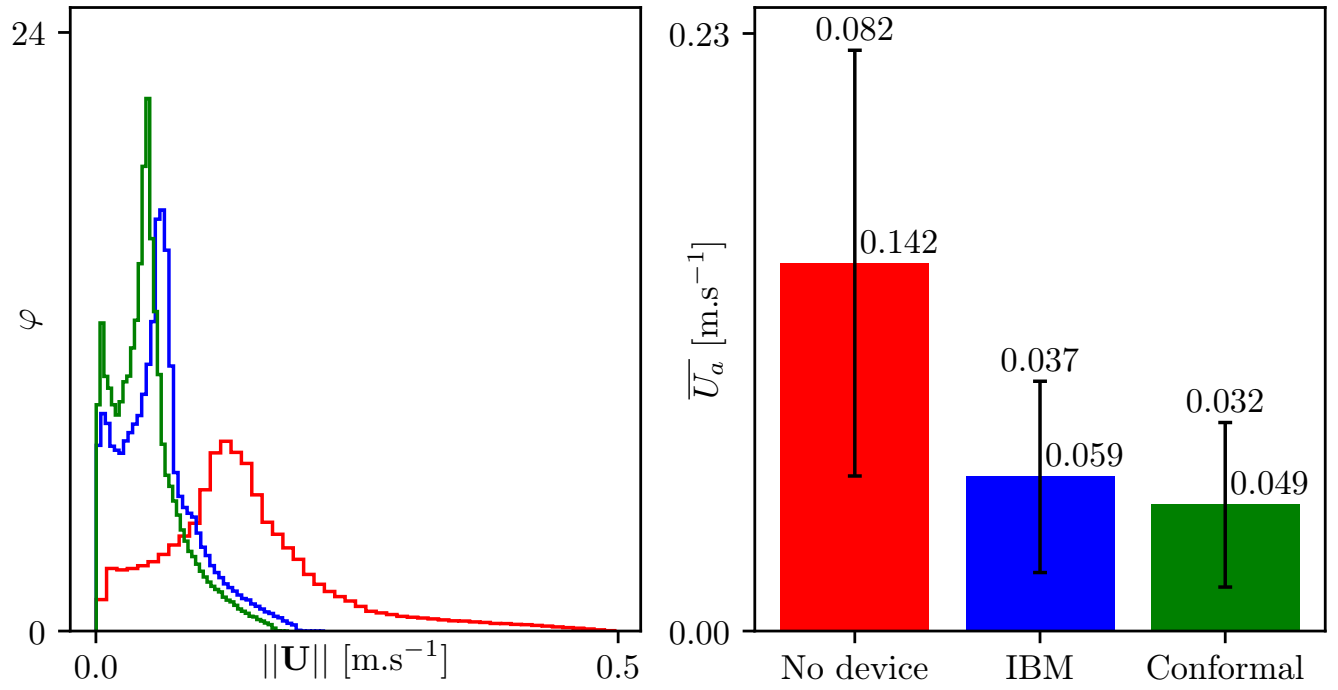

FIGURE 13 Quantitative results for the patient-specific set-up. Left: probability density function (PDF) $\varphi$ for the intra-saccular velocity magnitude. Right: Spatially averaged intra-saccular velocity magnitude given by Eq. 20 as well as standard deviation of the PDF for errors bars. Colors are the same as for the left part.

\begin{tabular}{lccc}
\hline & Edge-based IBM & Conformal & Reduction factor \\
\hline Total memory usage (in Gb) & 10.4 & 236.6 & 22.75 \\
$\mathrm{RCT}_{1}$ (in s $\cdot$ Nprocessors/Ncells/Niterations) & $4.03 \times 10^{-6}$ & $3.56 \times 10^{-6}$ & 0.88 \\
$\mathrm{RCT}_{2}$ (in s $\cdot$ Nprocessors) & $1.296 \times 10^{6}$ & $7.473 \times 10^{9}$ & 5766 \\
\hline
\end{tabular}

TABLE 2 Computational costs: memory usage and reduced computational time. The reduction factor column stands for conformal over IBM. 


\section{4 | DISCUSSION}

This study introduces a novel heterogeneous approach intended to numerically solve blood flow for intracranial aneurysms treated with endovascular devices such as flow-diverters. Mimicking the struts effects on the flow via drag forces regularized on the fluid mesh, this model endeavours to balance computational costs and low potential sources of errors while reproducing complex flow patterns near the wires. Several hypotheses regarding geometrical aspects and drag interactions between struts are formulated in order to build the model. More specifically, it is hypothesized that the overall device's effect on the flow stems from the independent superposition of drag forces generated by two sets of infinite parallel wires forming the device. Each set of wires is treated independently and interactions between wires are only modelled inside each set. Additionally, the sections where the two sets of cylinders merge together, herein referred to as 'crossings', are implicitly modelled as the sum of forces coming from each set. This means that this model is not adapted to very dense weaves, in which crossings cannot be neglected. The drag force is decomposed into several directions as a function of the geometry. For each component, the drag force model either comes directly from the literature or is inspired by existing drag laws, modified and calibrated with 3D CFD computations. It is also emphasized that the different expressions of the drag force components need the knowledge of an undisturbed infinity velocity $\mathbf{U}_{\infty}$, which cannot be simply defined in real cases. Therefore, a numerical strategy intended to reconstruct this quantity from available local flow measurements is developed, using both conservation principles and 2D CFD datas. The proposed heterogeneous model is validated with two configurations: 3D idealized and patient-specific, the conformal approach being considered as ground truth. Providing a good qualitative comparison of velocity fields and correctly representing the flow heterogeneities near the wires for both configurations, it is found that the maximum error reaches $59 \%$ for the idealized device. These error can be explained by an underestimation of downstream velocities in this situation, suggesting that the drag forces coming from the model are overestimated in comparison to the conformal ones. Conversely, it has been found that the drag forces are underestimated in the patient-specific case.

Comparison with the performances of homogeneous models is interesting, but should be led with caution, since boundary conditions, devices, aneurysmal geometries and meshes differ, as well as the metrics to assess the models. Note first that Raschi et al. [20] showed that their porous medium implementation systematically overestimates the effect of the device and thus underestimates flow-related quantities (WSS, velocities ...) in the aneurysm sac compared to the conformal results, which is the opposite of our model. To perform quantitative comparisons, Raschi et al. [20] used the reduction of any hemodynamic quantity $Q$ between no-device and device results such that $Q^{\text {porous }} / Q^{\text {no device }}$ and $Q^{\text {conformal }} / Q^{\text {no device }}$ are compared. They observed a maximum of $10 \%$ difference between porous and conformal reductions for an index equivalent to $\overline{U_{a}}$ (see Eq. 20 , , the average velocity inside the aneurysm. Computing the same reduction ratios in the aneurysm simulation presented, one obtains $\bar{U}_{a}$ heterogeneous $/ \bar{U}_{a}^{\text {no device }}=41.5 \%$ and ${\overline{U_{a}}}^{\text {conformal }} / \bar{U}_{a}^{\text {no device }}=34 \%$, which entails a $7.5 \%$ difference, smaller to the one obtained by Raschi et al. ${ }^{\text {[20] }}$. The overestimation of the device effect observed by Raschi et al. [20] is also retrieved in the porous model developed by Augsburger et al. [19], for the shear-driven aneurysms only. Although the errors given in Augsburger et al. [19] are not computed the same way as in the present work, the order of magnitude are the same with approximately $20 \%$ for intrasaccular velocities errors between porous and conformal methods. 2D steady computations performed with the screen model by $\mathrm{Li}$ et al. [59] exhibit intra-saccular velocities errors from $11 \%$ to $60 \%$ depending on the stent deployment and porosities, with a mean value around 30\% among all configurations, which is higher than the current 3D errors obtained on the patient-specific configuration in the present work. The 3D implementation of the aforementioned model inside three patient-specific geometries by Li et al. ${ }^{[22]}$ yielded overestimations and underestimations of intra-saccular velocities for $1 / 3$ and $2 / 3$ of the geometries, respectively. A direct comparison of errors with the work by Li et al. ${ }^{[22]}$ is not possible, since they assess the errors on the WSS at several points on the aneurysm surface. Nevertheless, the errors are of the same order of magnitude as in the present work.

Despite exhibiting similar errors with homogeneous methods, the heterogeneous model has demonstrated its capability to capture very detailed flow gradients near the device wires, which is impossible to obtain with other approaches. This makes the model highly versatile and capable of studying in details how wires compaction at the neck influences the intra-saccular environment. Compared to homogeneous models, another advantage is that the heterogeneous model results from well-identified assumptions and sub-models which can be revisited and improved. The proposed model could first be enhanced by taking into account more complex phenomena such as crossings effects and longitudinal interactions between struts. In particular, one could envision that the 3D conformal CFD computations used to calibrate the tangential component could be performed with more geometric and flow conditions, thereby enabling to parametrically study in details drag interactions for all three components of the forces on the cylinders. This introduces at least two additional parameters on which the drag forces could depend, namely the $\alpha_{\infty}^{l, t}$ angle and the inter-wire $\alpha$ angle, which has been fixed to $\pi / 2$ in this study. Therefore, this would bring the total number of 
relevant parameters to six, namely $\left(R e, w, D, \alpha, \alpha_{\infty}^{n, t}, \alpha_{\infty}^{l, t}\right)$, which entails a high computational burden. Additionally, performing this kind of 3D parametric study would give insights into the force exerted by the crossings sections and how they could be embedded into the edge-based IBM modelling. Another major improvement of the current edge-based model concerns the diffusive flow regime, which was intentionally not tackled in this work since it was considered to be critical for endovascular devices to reduce the strongest flow features coming from the parent artery. Specifically designing such a diffusive model would require to revisit the notion of wakes and to study long-range interactions between closely-packed cylinders, especially for the tangential and longitudinal components of the force. Combining such a model with the one presented in this work would be of course very desirable and could be useful to account for all flow conditions encountered by endovascular devices.

\section{5 | ACKNOWLEDGEMENTS}

The authors would like to gratefully thank Dr. Moureau and Dr. Lartigue (CORIA, UMR 6614) and the SUCCESS scientific group for providing the YALES2 code, which constitutes the basis of the YALES2BIO CFD solver. Dr. Siguenza is acknowledged for the numerical mechanical deployment of the flow-diverter. Simulations were performed using HPC resources from GENCI-CINES (Grant No. A0060307194 and A0080307194) and with the support of the High Performance Computing Platform MESO@LR, financed by the Occitanie / Pyrénées-Méditerranée Region, Montpellier Mediterranean Metropole and the University of Montpellier. We finally would like to thank the LabEx Numev (convention ANR-10-LABX-20) for supporting the YALES2BIO project.

\section{APPENDIX}

\section{A EDGE-BASED RKPM}

In the following, we detail the computation of the RKPM coefficients $\beta_{k}$ introduced in Eq. 16 in the case of edge-based IBM. To simplify the following derivations, the reasoning is made for one edge only since overall conservation is ensured by the summation over all edges given by Eq. 15

The main objective of the regularization step is to generate a volume source term $\mathbf{f}$ that faithfully represents the constant linear density of force $\mathbf{F}_{\mathbf{m}}$ along the $\mathrm{m}^{\text {th }}$ edge. Being inspired from nodal IBM considerations stated by Pinelli et al. ${ }^{[46]}$ and 
Mendez et al. [30] who used the RKPM principle introduced by Liu et al. [47], the main constraint for the source term is that the mathematical moments of $\mathbf{F}_{\mathbf{m}}$ are conserved up to a given order. For a second order method, this constraint is given by:

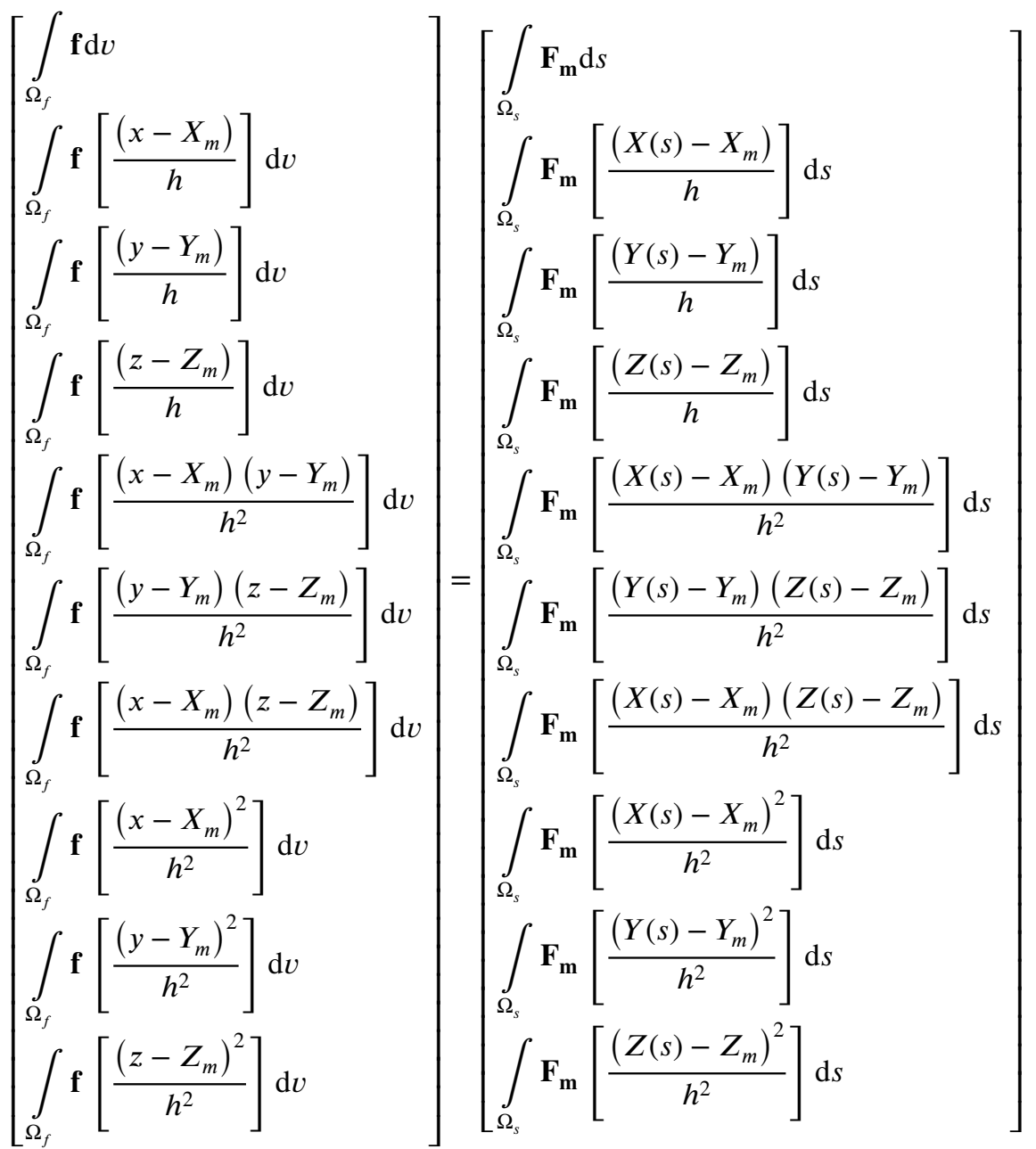

with $\mathbf{X}_{\mathbf{m}}=\left(X_{m}, Y_{m}, Z_{m}\right)$ the coordinates of the center of the $\mathrm{m}^{\text {th }}$ edge, $\mathbf{X}(\mathbf{s})=(X(s), Y(s), Z(s))$ the $\mathrm{m}^{\text {th }}$ edge coordinates parametrized by the curvilinear coordinate $s$ and $\mathbf{x}=(x, y, z)$ the coordinates of the mesh fluid node. The right-hand side of Eq. A1 represents the solid moments $\mathbf{M}_{\mathbf{s}}$, while the left-hand side stands for the fluid moments $\mathbf{m}_{\mathbf{f}}$. The first component of $\mathbf{M}_{\mathrm{s}}$ states that regularization must conserve the integral of the force over the edge. The next three components are linked to the mechanical moment of the force on the edge, which is zero by definition when being computed on the edge center $\mathbf{X}_{\mathbf{m}}$. 
Since the force density per unit of length $\mathbf{F}_{\mathbf{m}}$ is constant along the $\mathrm{m}^{\text {th }}$ edge, it can be taken out from the integral in Eq. A1 Therefore, $\mathbf{M}_{\mathbf{s}}$ only depends on the geometry of the edge and can be computed analytically such that:

$$
\mathbf{M}_{\mathbf{s}}=\mathbf{F}_{\mathbf{m}} \times\left[\begin{array}{c}
L \\
0 \\
0 \\
0 \\
\frac{L}{h^{2}}\left(\frac{\left(X_{2}-X_{1}\right)\left(Y_{2}-Y_{1}\right)}{3}+\frac{X_{1} Y_{2}+Y_{1} X_{2}}{2}-X_{m} Y_{m}\right) \\
\frac{L}{h^{2}}\left(\frac{\left(Y_{2}-Y_{1}\right)\left(Z_{2}-Z_{1}\right)}{3}+\frac{Y_{1} Z_{2}+Z_{1} Y_{2}}{2}-Y_{m} Z_{m}\right) \\
\frac{L}{h^{2}}\left(\frac{\left(X_{2}-X_{1}\right)\left(Z_{2}-Z_{1}\right)}{3}+\frac{X_{1} Z_{2}+Z_{1} X_{2}}{2}-X_{m} Z_{m}\right) \\
\frac{L}{h^{2}}\left(\frac{\left(X_{2}-X_{1}\right)^{2}}{3}+X_{1} X_{2}-X_{m}^{2}\right) \\
\frac{L}{h^{2}}\left(\frac{\left(Y_{2}-Y_{1}\right)^{2}}{3}+Y_{1} Y_{2}-Y_{m}^{2}\right) \\
\frac{L}{h^{2}}\left(\frac{\left(Z_{2}-Z_{1}\right)^{2}}{3}+Z_{1} Z_{2}-Z_{m}^{2}\right)
\end{array}\right] .
$$

$\mathbf{X}_{\mathbf{1 , 2}}=\left(X_{1,2}, Y_{1,2}, Z_{1,2}\right)$ are the end coordinates of the edge of length $L$ (see Fig. 6).

Using the definition of the fluid source term given by Eq. 15 with only one solid edge and simplifying on both sides by $\mathbf{F}_{\mathbf{m}}$, the equality of moments given by Eq. A1 can be expressed as:

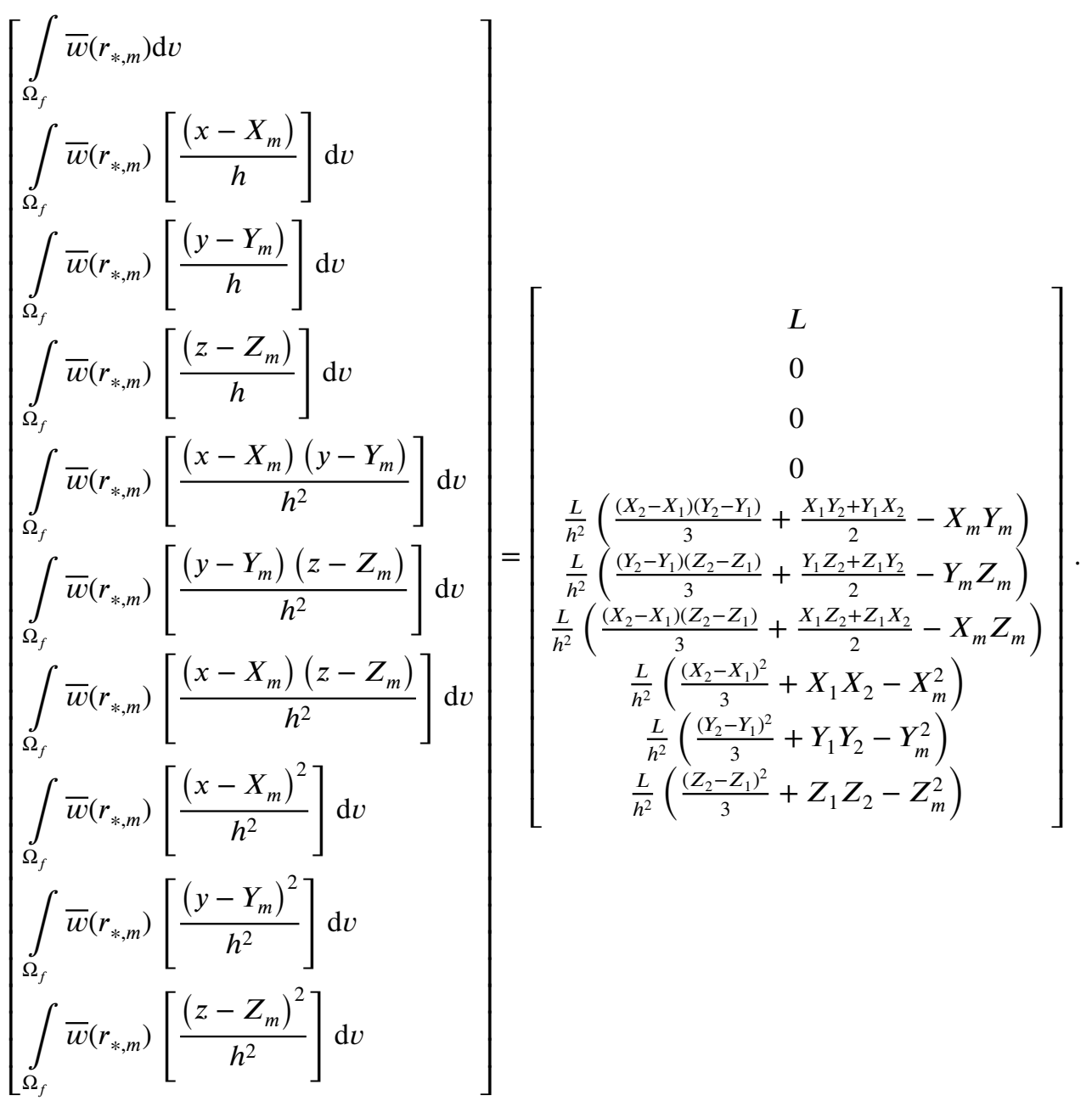


For the equality Eq. A3 to be satisfied on unstructured meshes, as intended here, we now write $\bar{w}\left(r_{*, m}\right)$ as a polynomial correction of the original window function $w\left(r_{*, m}\right)$ following the RKPM principle of Liu et al. ${ }^{47]}$ :

$$
\begin{aligned}
& \bar{w}\left(r_{*, m}\right)=w\left(r_{*, m}\right) \times\left[\beta_{0}+\beta_{1}\left(\frac{x-X_{m}}{h}\right)+\beta_{2}\left(\frac{y-Y_{m}}{h}\right)+\beta_{3}\left(\frac{z-Z_{m}}{h}\right)\right. \\
& +\beta_{4}\left(\frac{x-X_{m}}{h}\right)\left(\frac{y-Y_{m}}{h}\right)+\beta_{5}\left(\frac{x-X_{m}}{h}\right)\left(\frac{z-Z_{m}}{h}\right)+\beta_{6}\left(\frac{y-Y_{m}}{h}\right)\left(\frac{z-Z_{m}}{h}\right) \\
& \left.+\beta_{7}\left(\frac{x-X_{m}}{h}\right)^{2}+\beta_{8}\left(\frac{y-Y_{m}}{h}\right)^{2}+\beta_{9}\left(\frac{z-Z_{m}}{h}\right)^{2}\right],
\end{aligned}
$$

with $\beta_{k}$ the 10 unknowns coefficients of the correction polynomial. They are computed to satisfy the moments equality Eq. A3 For the next steps, it is convenient to introduce the moments $m_{a, b, c}$ of the original window function defined as:

$$
m_{a, b, c}=\int_{\Omega_{f}}\left(\frac{x-X_{m}}{h}\right)^{a}\left(\frac{y-Y_{m}}{h}\right)^{b}\left(\frac{z-Z_{m}}{h}\right)^{c} \times w\left(r_{*, m}\right) \mathrm{d} v .
$$

Using the linearity of the integral operator and the definition of $m_{a, b, c}$ given by Eq. A5 we end up with:

$$
\mathcal{M} \beta=\overline{\mathbf{M}}_{\mathrm{s}},
$$

with:

$$
\mathcal{M}=\left[\begin{array}{llllllllll}
m_{0,0,0} & m_{1,0,0} & m_{0,1,0} & m_{0,0,1} & m_{1,1,0} & m_{1,0,1} & m_{0,1,1} & m_{2,0,0} & m_{0,2,0} & m_{0,0,2} \\
m_{1,0,0} & m_{2,0,0} & m_{1,0,1} & m_{1,1,0} & m_{2,1,0} & m_{2,0,1} & m_{1,1,1} & m_{3,0,0} & m_{1,2,0} & m_{1,0,2} \\
m_{0,1,0} & m_{1,1,0} & m_{0,2,0} & m_{0,1,1} & m_{1,2,0} & m_{1,1,1} & m_{0,2,1} & m_{2,1,0} & m_{0,3,0} & m_{0,1,2} \\
m_{0,0,1} & m_{1,0,1} & m_{0,1,1} & m_{0,0,2} & m_{1,1,1} & m_{1,0,2} & m_{0,1,2} & m_{2,0,1} & m_{0,2,1} & m_{0,0,3} \\
m_{1,1,0} & m_{2,1,0} & m_{1,2,0} & m_{1,1,1} & m_{2,2,0} & m_{2,1,1} & m_{1,2,1} & m_{3,1,0} & m_{1,3,0} & m_{1,1,2} \\
m_{0,1,1} & m_{1,1,1} & m_{0,2,1} & m_{0,1,2} & m_{1,2,2} & m_{1,1,2} & m_{0,2,2} & m_{2,1,1} & m_{0,3,1} & m_{0,1,3} \\
m_{1,0,1} & m_{2,0,1} & m_{1,1,1} & m_{1,0,2} & m_{2,1,1} & m_{2,0,2} & m_{1,1,2} & m_{3,0,1} & m_{1,2,1} & m_{1,0,3} \\
m_{2,0,0} & m_{3,0,0} & m_{2,1,0} & m_{2,0,1} & m_{3,1,0} & m_{3,0,1} & m_{2,1,1} & m_{4,0,0} & m_{2,2,0} & m_{2,0,2} \\
m_{0,2,0} & m_{1,2,0} & m_{0,3,0} & m_{0,2,1} & m_{1,3,0} & m_{1,2,1} & m_{0,3,1} & m_{2,2,0} & m_{0,4,0} & m_{0,2,2} \\
m_{0,0,2} & m_{1,0,2} & m_{0,1,2} & m_{0,0,3} & m_{1,1,2} & m_{1,0,3} & m_{0,1,3} & m_{2,0,2} & m_{0,2,2} & m_{0,0,4}
\end{array}\right], \boldsymbol{\beta}=\left[\begin{array}{c}
\beta_{0} \\
\beta_{1} \\
\beta_{2} \\
\beta_{3} \\
\beta_{4} \\
\beta_{5} \\
\beta_{6} \\
\beta_{7} \\
\beta_{8} \\
\beta_{9}
\end{array}\right],
$$

and $\overline{\mathbf{M}}_{\mathrm{s}}$ the right hand side of Eq. A3 Inverting matrix $\mathcal{M}$, one can find all the $\beta_{k}$ coefficients as:

$$
\boldsymbol{\beta}=\mathcal{M}^{-1} \overline{\mathbf{M}_{\mathbf{s}}} .
$$

Since solid and fluid domains do not change over time, i.e. the device is fixed and the grid is not re-meshed, Eq. A8 is solved once for all at the start of the computation and the $\beta_{k}$ coefficients are stored to be used for the regularization step, enabling a low computational cost. It should be kept in mind that the $\beta_{k}$ coefficients are computed for each edge using Eq. A8. Concerning units, the components of $\boldsymbol{\beta}$ are equivalent to $\left[\mathrm{m}^{-2}\right]$ so as to be compatible with the ones in Eq. 15 which are [N.m $\left.\mathrm{m}^{-3}\right],\left[\mathrm{N} \cdot \mathrm{m}^{-1}\right.$ ] and [1] for $\mathbf{f}_{\mathbf{i}}, \mathbf{F}_{\mathbf{m}}$ and $w\left(r_{*, m}\right)$ respectively. Using these coefficients, the modified window $\bar{w}$ is used to compute the regularized volume source term $\mathbf{f}_{\mathbf{i}}$ at each $\mathrm{i}^{\text {th }}$ point of the fluid mesh as:

$$
\mathbf{f}_{\mathbf{i}}=\sum_{m=1}^{M} \mathbf{F}_{\mathbf{m}} \bar{w}\left(r_{i, m}\right) .
$$

Due to the summation, a fluid mesh point that lies within the support function of multiple edges receives contributions from all these edges. This is notably the case near the intersection points of the edges (see Fig. 6).

\section{B 3D CALIBRATION OF THE TANGENTIAL MODEL}

As already stated in subsubsection 2.1.3, there remains two unknowns in the tangential drag model corresponding to Algorithm 1: $N_{w}$ and $q_{p}$. The first one is needed to compute the width $\hat{w}=N_{w} D$ used to determine if cylinders are interacting, 
while the second one represents the homogeneous decay rate when cylinders are subjected to a purely tangential incoming flow (see Eq. 12). Values of these parameters were adjusted in order to best represent 3D CFD results obtained with the geometry depicted by Fig. 7 and for all the operating conditions given in Tab. 1 except $R e=2.5$, since the model is intended to be used for convective flow regimes only. A least square fitting procedure using the tangential forces generated by the fluid onto each of the two cylinder families coming from these conformal, well-resolved CFD simulations was performed.

Comparison of the fitted tangential model with the CFD data is shown in Fig. B1 for all the studied Reynolds numbers and for only one cylinder family. It has been found that $N_{w}=12$ and $q_{p}=0.5$ produce a behaviour similar to 3D CFD data. More precisely, it reproduces both the increase and decrease of drag force when the incoming flow goes from purely normal $\left(\alpha_{\infty}^{n, t}=\pi / 2\right)$ to tangential $\left(\alpha_{\infty}^{n, t}=0\right)$, this for various $W / D$ ratios and $\alpha_{\infty}^{l, t}$ values. It has been found that in general, the fitted tangential model goes from overestimating to underestimating forces when the flow becomes purely normal. This underestimation is even more pronounced when $\alpha_{\infty}^{l, t}=\pi / 3$.
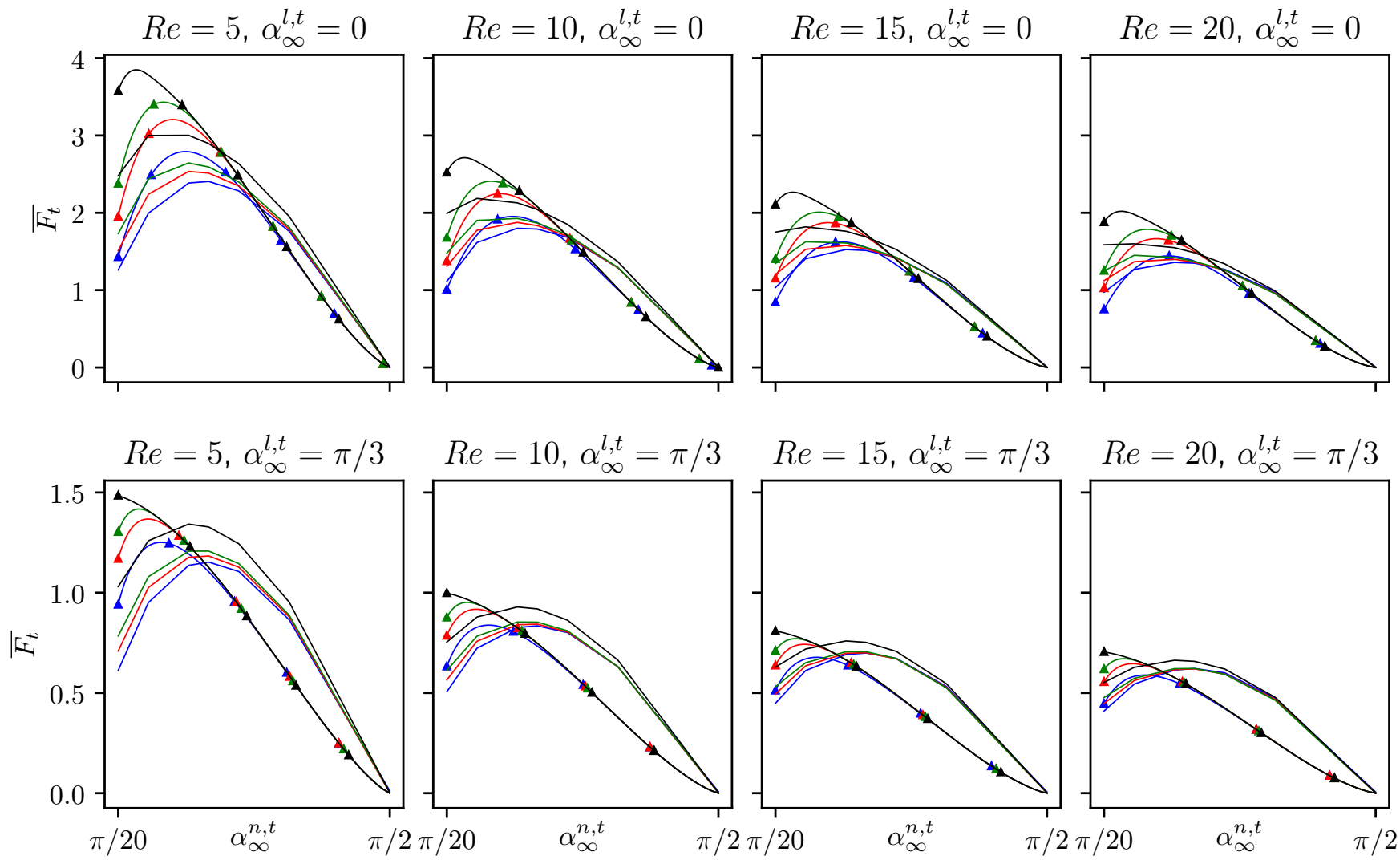

FIGURE B1 Tangential drag force model Algorithm 1 ( $\triangle$ symbols) and CFD 3D datas (solid lines) when incoming flow angles are $\alpha_{\infty}^{n, t} \in[\pi / 20, \pi / 2], \alpha_{\infty}^{l, t}=0$ (top row) and $\alpha_{\infty}^{l, t}=\pi / 3$ (bottom row). Several $W / D$ ratios are depicted: 6.0 (一), 8.0 (一), $10.0\left(\right.$ ( ) and $20.0($ ( ) $) .{ }_{F_{t}}^{\infty}$ is defined as $\overline{F_{t}}=\frac{{ }_{\text {F.t }}}{0.5 \rho|| \mathbf{U}_{\infty} \|^{2} D}$ 


\section{References}

[1] K. N. Kayembe, M. Sasahara, and F. Hazama. Cerebral aneurysms and variations in the circle of willis. Stroke, 15(9): $846-850,1984$. .

[2] G. J. E. Rinkel, M. Djibuti, A. Algra, and J. van Gijn. Prevalence and risk of rupture of intracranial aneurysms: A systematic review. Stroke, 29(1):251-256, 1998. .

[3] M. H. Vlak, A. Algra, R. Brandenburg, and G. J. Rinkel. Prevalence of unruptured intracranial aneurysms, with emphasis on sex, age, comorbidity, country, and time period: a systematic review and meta-analysis. The Lancet Neurology, 10(7): 626-636, 2011. .

[4] D. J. Nieuwkamp, L. E. Setz, A. Algra, F. H. Linn, N. K. de Rooij, and G. J. Rinkel. Changes in case fatality of aneurysmal subarachnoid haemorrhage over time, according to age, sex, and region: a meta-analysis. The Lancet Neurology, 8(7): 635-642, 2009. .

[5] R. D. Perrone, A. M. Malek, and T. Watnick. Vascular complications in autosomal dominant polycystic kidney disease. Nature Reviews Nephrology, 11(8):589-598, 2015. .

[6] J. K. Campos, B. Cheaney II, B. V. Lien, D. A. Zarrin, C. D. Vo, G. P. Colby, L.-M. Lin, and A. L. Coon. Advances in endovascular aneurysm management: flow modulation techniques with braided mesh devices. Stroke and Vascular Neurology, 5(3):1-13, 2020. .

[7] R. Kadirvel, Y.-H. Ding, D. Dai, I. Rezek, D. A. Lewis, and D. F. Kallmes. Cellular mechanisms of aneurysm occlusion after treatment with a flow diverter. Radiology, 270(2):394-399, 2014. .

[8] K. Ravindran, M. M. Salem, A. Y. Alturki, A. J. Thomas, C. S. Ogilvy, and J. M. Moore. Endothelialization following flow diversion for intracranial aneurysms: A systematic review. American Journal of Neuroradiology, 40(1):295-301, 2019. .

[9] M. N. Ngoepe, A. F. Frangi, J. V. Byrne, and Y. Ventikos. Thrombosis in cerebral aneurysms and the computational modeling thereof: A review. Frontiers in Physiology, 9:306, 2018.

[10] A. Rouchaud, C. Ramana, W. Brinjikji, Y.-H. Ding, D. Dai, T. Gunderson, J. Cebral, D. F. Kallmes, and R. Kadirvel. Wall apposition is a key factor for aneurysm occlusion after flow diversion: A histologic evaluation in 41 rabbits. American Journal of Neuroradiology, 37(7):2087-2091, 2016. .

[11] T. Becske, D. F. Kallmes, I. Saatci, C. G. McDougall, I. Szikora, G. Lanzino, C. J. Moran, H. H. Woo, D. K. Lopes, A. L. Berez, D. J. Cher, A. H. Siddiqui, E. I. Levy, F. C. Albuquerque, D. J. Fiorella, Z. Berentei, M. Marősfoi, S. H. Cekirge, and P. K. Nelson. Pipeline for uncoilable or failed aneurysms: Results from a multicenter clinical trial. Radiology, 267 (6):858-868, 2013. .

[12] Z. Kulcsár, E. Houdart, A. Bonafé, G. Parker, J. Millar, A. J. P. Goddard, S. Renowden, G. Gál, B. Turowski, K. Mitchell, F. Gray, M. Rodriguez, R. van den Berg, A. Gruber, H. Desal, I. Wanke, and D. A. Rüfenacht. Intra-aneurysmal thrombosis as a possible cause of delayed aneurysm rupture after flow-diversion treatment. American Journal of Neuroradiology, 32 (11):20-25, 2011. .

[13] W. Brinjikji, M. H. Murad, G. Lanzino, H. J. Cloft, and D. F. Kallmes. Endovascular treatment of intracranial aneurysms with flow diverters: A meta-analysis. Stroke, 44(2):442-447, 2013. .

[14] R. Ouared, I. Larrabide, O. Brina, P. Bouillot, G. Erceg, H. Yilmaz, K. O. Lovblad, and V. Mendes Pereira. Computational fluid dynamics analysis of flow reduction induced by flow-diverting stents in intracranial aneurysms: A patient-unspecific hemodynamics change perspective. Journal of NeuroInterventional Surgery, 8:1288-1293, 2016. .

[15] I. Larrabide, A. J. Geers, H. G. Morales, M. L. Aguilar, and D. A. Rüfenacht. Effect of aneurysm and ica morphology on hemodynamics before and after flow diverter treatment. Journal of NeuroInterventional Surgery, 7:272-280, 2014. . 
[16] F. Mut, M. Raschi, E. Scrivano, C. Bleise, J. Chudyk, R. Ceratto, P. Lylyk, and J. R. Cebral. Association between hemodynamic conditions and occlusion times after flow diversion in cerebral aneurysms. Journal of NeuroInterventional Surgery, 7:286-290, 2015. .

[17] G. Janiga, C. Rössl, M. Skalej, and D. Thévenin. Realistic virtual intracranial stenting and computational fluid dynamics for treatment analysis. Journal of Biomedical Optics, 46:7-12, 2013. .

[18] T. W. Peach, D. Ricci, and Y. Ventikos. A virtual comparison of the eclips device and conventional flow-diverters as treatment for cerebral bifurcation aneurysms. Cardiovascular Engineering and Technology, (7), 2019. .

[19] L. Augsburger, P. Reymond, D. A. Rufenacht, and N. Stergiopulos. Intracranial stents being modeled as a porous medium: Flow simulation in stented cerebral aneurysms. Annals of Biomedical Engineering, 39(11):850-863, 2010. .

[20] M. Raschi, F. Mut, R. Löhner, and J. R. Cebral. Strategy for modeling flow diverters in cerebral aneurysms as a porous medium. International Journal for Numerical Methods in Biomedical Engineering, 30(3):909-925, 2014. .

[21] S. Li, J. Latt, and B. Chopard. Model for pressure drop and flow deflection in the numerical simulation of stents in aneurysms. International Journal for Numerical Methods in Biomedical Engineering, 34(3):e2949, 2018. .

[22] S. Li, B. Chopard, and J. Latt. Continuum model for flow diverting stents in 3d patient-specific simulation of intracranial aneurysms. Journal of Computational Science, 38(11):101045, 2019. .

[23] G. Janiga, L. Daróczy, P. Berg, D. Thévenin, M. Skalej, and O. Beuing. An automatic cfd-based flow diverter optimization principle for patient-specific intracranial aneurysms. Journal of Biomedical Optics, 48(14):3846-3852, 2015. .

[24] R. J. Damiano, V. M. Tutino, N. Paliwal, D. Ma, J. M. Davies, A. H. Siddiqui, and H. Meng. Compacting a single flow diverter versus overlapping flow diverters for intracranial aneurysms: A computational study. American Journal of Neuroradiology, 38(1):603-610, 2017. .

[25] M. Zhang, Y. Li, X. Zhao, D. I. Verrelli, W. Chong, M. Ohta, and Y. Qian. Haemodynamic effects of stent diameter and compaction ratio on flow-diversion treatment of intracranial aneurysms: A numerical study of a successful and an unsuccessful case. Journal of Biomechanics, 58:179-186, 2017. .

[26] J. Xiang, D. Ma, K. V. Snyder, E. I. Levy, A. H. Siddiqui, and H. Meng. Increasing flow diversion for cerebral aneurysm treatment using a single flow diverter. Neurosurgery, 75:286-294, 2014. .

[27] K. J. Chodzyǹski, P. Uzureau, V. Nuyens, A. Rousseau, G. Coussement, and K. Zouaoui Boudjeltia. The impact of arterial flow complexity on flow diverter outcomes in aneurysms. Scientific Reports, 10(6), 2020. .

[28] C. S. Peskin. The immersed boundary method. Acta Numerica, 11:479-517, 2002.

[29] V. Moureau, P. Domingo, and L. Vervisch. Design of a massively parallel CFD code for complex geometries. Comptes Rendus Mécanique, 339(2-3):141-148, 2011.

[30] S. Mendez, E. Gibaud, and F. Nicoud. An unstructured solver for simulations of deformable particles in flows at arbitrary Reynolds numbers. Journal of Computational Physics, 256(1):465-483, 2014.

[31] J. Sigüenza, S. Mendez, and F. Nicoud. How should the optical tweezers experiment be used to characterize the red blood cell membrane mechanics? Biomechanics and Modeling in Mechanobiology, 16:1645-1657, 2017.

[32] L. Lanotte, J. Mauer, S. Mendez, D. A. Fedosov, J.-M. Fromental, V. Claveria, F. Nicoud, G. Gompper, and M. Abkarian. Red cells' dynamic morphologies govern blood shear thinning under microcirculatory flow conditions. Proceedings of the National Academy of Sciences USA, 113(47):13289-13294, 2016. .

[33] J. Mauer, S. Mendez, L. Lanotte, F. Nicoud, M. Abkarian, G. Gompper, and D. A. Fedosov. Flow-induced transitions of red blood cell shapes under shear. Physical Review Letters, 121(118103), 2018.

[34] C. Iss, D. Midou, A. Moreau, D. Held, A. Charrier, S. Mendez, A. Viallat, and E. Helfer. Self-organization of red blood cell suspensions under confined 2d flows. Soft Matter, 15:2971-2980, 2019. 
[35] P. Taraconat, J.-P. Gineys, D. Isèbe, F. Nicoud, and S. Mendez. Numerical simulation of deformable particles in a Coulter counter. International Journal for Numerical Methods in Biomedical Engineering, 35(11):e3243, 2019. .

[36] V. Zmijanovic, S. Mendez, V. Moureau, and F. Nicoud. About the numerical robustness of biomedical benchmark cases: Interlaboratory FDA's idealized medical device. International Journal for Numerical Methods in Biomedical Engineering, 33(1):e02789:1-17, 2017.

[37] T. Puiseux, A. Sewonu, O. Meyrignac, H. Rousseau, F. Nicoud, S. Mendez, and R. Moreno. Reconciling PC-MRI and CFD: an in-vitro study. NMR in Biomedicine, 2019.

[38] C. Chnafa, S. Mendez, and F. Nicoud. Image-based large-eddy simulation in a realistic left heart. Computers and Fluids, 94:173-187, 2014.

[39] C. Chnafa, S. Mendez, and F. Nicoud. Image-based simulations show important flow fluctuations in a normal left ventricle: What could be the implications? Annals of Biomedical Engineering, 44(11):3346-3358, 2016.

[40] M. Malandain, N. Maheu, and V. Moureau. Optimization of the deflated conjugate gradient algorithm for the solving of elliptic equations on massively parallel machines. Journal of Computational Physics, 238:32-47, 2013.

[41] P. Bouillot, O. Brina, R. Ouared, H. Yilmaz, M. Farhat, G. Erceg, K.-O. Lovblad, M. I. Vargas, Z. Kulcsar, and V. M. Pereira. Geometrical deployment for braided stent. Medical Image Analysis, 30(5):85-94, 2016. .

[42] T. Müller, J. Meyer, and G. Kasper. Low reynolds number drag and particle collision efficiency of a cylindrical fiber within a parallel array. Journal of the Aeronautical Sciences, 77:50-66, 2014. . URL http://dx.doi.org/10.1016/j.jaerosci.2014. 07.007.

[43] T. Miyagi. Viscous flow at low reynolds numbers past an infinite row of equal circular cylinders. Journal of the Physical Society of Japan, 13(5):493-496, 1958. .

[44] D. G. Crowdy. Uniform flow past a periodic array of cylinders. European Journal of Mechanics - B/Fluids, 56(3):120-129, 2016. .

[45] N. Marheineke and R. Wegener. Modeling and application of a stochastic drag for fibers in turbulent flows. International Journal of Multiphase Flow, 37(3):136-148, 2011. .

[46] A. Pinelli, I. Z. Naqavi, U. Piomelli, and J. Favier. Immersed-boundary methods for general finite-difference and finitevolume Navier-Stokes solvers. Journal of Computational Physics, 229:9073-9091, 2010.

[47] W. K. Liu, S. Jun, and Y. F. Zhang. Reproducing kernel particle methods. International Journal for Numerical Methods in Fluids, 20:1081-1106, 1995.

[48] J. Sigüenza, S. Mendez, D. Ambard, F. Dubois, F. Jourdan, R. Mozul, and F. Nicoud. Validation of an immersed thick boundary method for simulating fluid-structure interactions of deformable membranes. Journal of Computational Physics, 322:723-746, 2016.

[49] C. Geuzaine and J.-F. Remacle. Gmsh: A 3-d finite element mesh generator with built-in pre- and post-processing facilities. International Journal for Numerical Methods in Engineering, 79(9):1309-1331, 2009. .

[50] Wi. Schroeder, K. Martin, and B. Lorensen. The Visualization Toolkit: An Object-Oriented Approach To 3D Graphics. Kitware Inc., 4.1 edition, July 2018.

[51] A. Utkarsh. The ParaView Guide: Community Edition. Kitware Inc., June 2017.

[52] W. E. Lorensen and H. E. Cline. Marching cubes: A high resolution 3d surface construction algorithm. ACM SIGGRAPH Computer Graphics, 21(8):163-169, 1987. .

[53] L. Antiga, M. Piccinelli, L. Botti, B. Ene-Iordache, A. Remuzzi, and D. A. Steinman. An image-based modeling framework for patient-specific computational hemodynamics. Medical \& Biological Engineering \& Computing, 46(11):1097-1112, November 2008. . URL http://link.springer.com/10.1007/s11517-008-0420-1 
[54] C. Dapogny, C. Dobrzynski, and P. Frey. Three-dimensional adaptive domain remeshing, implicit domain meshing, and applications to free and moving boundary problems. Journal of Computational Physics, 262(4):358-378, 2014 . .

[55] K. Valen-Sendstad, M. Piccinelli, R. KrishnankuttyRema, and D. A. Steinman. Estimation of inlet flow rates for imagebased aneurysm cfd models: Where and how to begin? Annals of Biomedical Engineering, 43(6):1422-1431, june 2015. . URL http://link.springer.com/10.1007/s10439-015-1288-5

[56] C. Chnafa, P. Bouillot, O. Brina, B. M. A. Delattre, M. I. Vargas, K. O. Lovblad, V. M. Pereira, and D. A. Steinman. Vessel calibre and flow splitting relationships at the internal carotid artery terminal bifurcation. Physiological Measurement, 38 (11):2044-2057, 2017. .

[57] Y. Hoi, B. A. Wasserman, Y. J. Xie, S. S. Najjar, L. Ferruci, E. G. Lakatta, G. Gerstenblith, and D. A Steinman. Characterization of volumetric flow rate waveforms at the carotid bifurcations of older adults. Physiological Measurement, 31(3):291-302, March 2010. . URL http://stacks.iop.org/0967-3334/31/i=3/a=002?key=crossref. d0c2fda4d23842496e89dc93a8607322.

[58] C. Chnafa, P. Bouillot, O. Brina, M. Najafi, B. M. A. Delattre, M. I. Vargas, V. M. Pereira, and D. A. Steinman. Errors in power-law estimations of inflow rates for intracranial aneurysm cfd. Journal of Biomechanics, 80(10):159-165, 2018. .

[59] S. Li, J. Latt, and B. Chopard. The application of the screen-model based approach for stents in cerebral aneurysms. Computers and Fluids, 172(8):651-660, 2018. . 